\title{
Study on the Deformation and Stress Analysis of the Sponge/Rubber Adapter
}

\author{
Jianlin Zhong $\mathbb{D}^{\mathbb{D}}$, Jie Ren $(\mathbb{D}$, and Dawei Ma \\ School of Mechanical Engineering, Nanjing University of Science and Technology, Nanjing 210094, China \\ Correspondence should be addressed to Jie Ren; renjie@njust.edu.cn
}

Received 6 February 2018; Revised 28 April 2018; Accepted 9 May 2018; Published 10 July 2018

Academic Editor: Alkis S. Paipetis

Copyright (C) 2018 Jianlin Zhong et al. This is an open access article distributed under the Creative Commons Attribution License, which permits unrestricted use, distribution, and reproduction in any medium, provided the original work is properly cited.

\begin{abstract}
The analysis of adaptors' deformation and stress is an important prerequisite for the design of the adapters. This paper proposed the mathematical description and analytical method for the sponge cylinder and rubber cylinder under pressure. The analytical formulas on deformation and stress of interference fit between adapters and launchers are deduced. Also, the correctness of the analytical formula is verified by numerical simulation. Furthermore, the effect of precompression and the sponge's thickness ratio on adapter's deformation and stress is studied. The following results can be concluded: the results of numerical simulation are basically consistent with that of analytical formula. After installing the adapter into the launcher, the rubber layer will move inward as a whole, then the sponge layer and the rubber layer are subjected to radial and tangential compressive stress. When the precompression is smaller than 0.0075 or larger than 0.0015 , the deformation and stress of adapter are approximately in proportion to precompression. If the precompression keeps constant, the deformation and stress of adapter will have extremum when the thickness ratio of sponge equals to a certain value.
\end{abstract}

\section{Introduction}

In the cold launching system, there are elastic cushions for adapters between the missile and the metal cylinder. The adapter is usually glued by the sponge cylinder and the rubber cylinder [1-3], as shown in Figure 1. During the ejection of the missile, the friction between the adapter and the launcher could reduce the ejection height of the missile and is determined by the radial stress caused by the adapter's compression deformation [4]. Therefore, the study of adapters' deformation and stress has an important engineering application value for the design of adapters.

As the adapter's deformation and stress analytical research is based on the study on sponge cylinder and the rubber cylinder, many studies have been conducted in recent years. The modeling of mechanical characteristics of sponge material is studied by Koohbor B et al. [5-7] and is verified with experiments. Liang et al. [8] simulate the damping performance of the sponge components using the neural network theory. Based on the study on identification process of mechanical properties of rubber materials, the constitutive equations for small strain and large strain are proposed by $\mathrm{Hao} \mathrm{H}$ et al. $[9,10]$. Lee $\mathrm{H}$ S et al. [11, 12] studied the deformation characteristics of rubber cylinders subjected to torsion, axial force, radial force, and skew load. The radial stiffness formula of rubber cylinder is derived by Horton J M [13], and the theoretical analytical formula and experimental data are compared and verified. $\mathrm{Yu} \mathrm{Z}$ et al. $[14,15]$ give an analytical solution for the interference fit between superelastic incompressible rubber and linear elastic material under a certain plane strain condition. Nevertheless, there are few reports on the stress and deformation modes of the sponge/rubber adapters.

In this paper, the interference fit between the adapter and the launcher is simplified as an axisymmetric plane strain problem. Based on the Blatz-Ko strain energy function of the sponge material and the three time reduced polynomial constitutive model of rubber material, the differential equation description and the plane axisymmetric analytical solution of the sponge cylinder and the rubber cylinder are proposed. Then, the analytical formulas on deformation and stress of interference fit between adapters and launchers are deduced and the correctness of the analytical formula is verified by numerical simulation. Furthermore, the effect of 


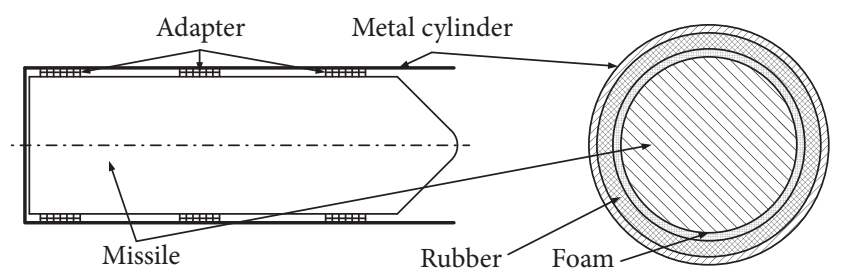

FIGURE 1: Schematic diagram of the launcher and the adapter.

precompression and the sponge's thickness ratio on adapter's deformation and stress is studied. The research methods and results in this paper can provide reference for the design of adapters

\section{Mathematical Description of Sponge Cylinder under Compression}

2.1. Differential Equation Description of Sponge Cylinder. According to the study of Ju et al. [16], Poisson's ratio of sponge is approximately 0 when pressed, assuming that the load and boundary conditions are axisymmetric, it can be simplified as an axisymmetric plane strain problem. In the initial stage, the inner and out radius of sponge cylinder are, respectively, $R_{I}$ and $R_{O}$, and then turn into $r_{I}$ and $r_{O}$ after deformation under the action of inner pressure of $\sigma_{r I}$ and out pressure of $\sigma_{r O}$. The cylindrical coordinate system is adopted with the material coordinate of $(R, \Theta, Z)$ and the spatial coordinate of $(r, \theta, z)$. Because of the axisymmetric plane strain problem, the deformation mode can be set as

$$
\begin{aligned}
& r=f(R), \\
& \theta=\Theta, \\
& z=\lambda Z
\end{aligned}
$$

where $\lambda$ is the elongation of the cylinder in $z$ direction.

According to (1), the main elongations of the deformation gradient are

$$
\begin{aligned}
& \lambda_{r}=\frac{d r}{d R}, \\
& \lambda_{\theta}=\frac{r}{R}, \\
& \lambda_{z}=1
\end{aligned}
$$

Sponge and rubber can produce large nonlinear deformation. The strain energy function is usually used to describe the deformation properties of the material [14]. Sponge is a hyperelastic compressible material whose strain energy function $W$ is a function of 3 main elongations, namely, $W=$ $W\left(\lambda_{r}, \lambda_{\theta}, \lambda_{z}\right)$. The Cauchy stress in the 3 main elongation directions is [17]

$$
\begin{gathered}
\sigma_{r}=\frac{1}{\lambda_{\theta} \lambda_{z}} \frac{\partial W}{\partial \lambda_{r}}, \\
\sigma_{\theta}=\frac{1}{\lambda_{r} \lambda_{z}} \frac{\partial W}{\partial \lambda_{\theta}},
\end{gathered}
$$

$$
\sigma_{z}=\frac{1}{\lambda_{r} \lambda_{\theta}} \frac{\partial W}{\partial \lambda_{z}}
$$

The axisymmetric plane strain problem should satisfy the static equilibrium equation with ignorance of gravity [18].

$$
\frac{d \sigma_{r}}{d r}+\frac{1}{r}\left(\sigma_{r}-\sigma_{\theta}\right)=0
$$

Equation (3) is substituted into (4):

$$
R \frac{\partial \lambda_{r}}{\partial R} \frac{\partial^{2} W}{\partial \lambda_{r}^{2}}+\frac{\partial^{2} W}{\partial \lambda_{r} \partial \lambda_{\theta}}\left(\lambda_{r}-\lambda_{\theta}\right)+\frac{\partial W}{\partial \lambda_{r}}-\frac{\partial W}{\partial \lambda_{\theta}}=0
$$

At present, the strain energy functions for compressible materials mainly consist of Ogden-Hill and Blatz-Ko strain energy function. The Blatz-Ko strain energy function has a simple form and can describe the deformation characteristics of compressible sponge material, and its strain energy function is [19]

$$
W\left(\lambda_{r}, \lambda_{\theta}, \lambda_{z}\right)=\frac{\mu}{2}\left(\lambda_{r}^{-2}+\lambda_{\theta}^{-2}+\lambda_{z}^{-2}+2 \lambda_{r} \lambda_{\theta} \lambda_{z}-5\right)
$$

where $\mu$ is the shear modulus of sponge in a natural state.

Equation (6) is substituted into (5):

$$
3 R \lambda_{r}^{\prime}+\left(\frac{\lambda_{r}^{4}}{\lambda_{\theta}^{3}}-\lambda_{r}\right)=0
$$

where $\lambda_{r}^{\prime}=d \lambda_{r} / d R$.

2.2. Differential Equation Analysis. Set $t=\lambda_{r} / \lambda_{\theta}=R r^{\prime} / r$, and then take derivative of $\mathrm{R}$ to obtain

$$
R \lambda_{r}^{\prime}=\lambda_{\theta} R t^{\prime}-\lambda_{r}+\frac{\lambda_{r}^{2}}{\lambda_{\theta}}
$$

The expression of intermediate variable $t$ and (8) are substituted into (7):

$$
\begin{aligned}
& \frac{d R}{R}=\frac{3 d t}{\left(-t^{4}-3 t^{2}+4 t\right)} \\
& \frac{d r}{r}=-\frac{3 d t}{\left(t^{3}+3 t-4\right)}
\end{aligned}
$$

The integral solution is applied on the ordinary differential equations (9), and the deformation function of the sponge cylinder can be obtained.

$$
\begin{aligned}
& R^{8}=\frac{A_{0} t^{6} e^{(6 / \sqrt{45}) \tan ^{-1}((2 t+1) / \sqrt{15})}(1-t)^{-4}}{\left(t^{2}+t+4\right)} \\
& r^{4}=\frac{A_{0} t^{6} e^{(6 / \sqrt{45}) \tan ^{-1}((2 t+1) / \sqrt{15})}\left(t^{2}+t+4\right)}{(1-t)^{2}}
\end{aligned}
$$

where $A_{0}$ and $B_{0}$ are integral constant and are related to boundary conditions. 
The Cauchy stress in three directions could be obtained by combining (3) and (6).

$$
\begin{aligned}
& \sigma_{r}=\mu\left(1-\frac{1}{\lambda_{r}^{3} \lambda_{\theta}}\right), \\
& \sigma_{\theta}=\mu\left(1-\frac{1}{\lambda_{\theta}^{3} \lambda_{r}}\right), \\
& \sigma_{z}=\mu\left(1-\frac{1}{\lambda_{r} \lambda_{\theta}}\right)
\end{aligned}
$$

2.3. Dimensionless of Deformation and Stress. $\eta=R / R_{0}$ represents the dimensionless radius before deformation, $\xi=$ $r / R_{O}$ represents dimensionless radius after deformation and $\xi_{i}=\sigma_{i} / \mu(i=r, \theta, z)$ is the dimensionless stress, $A=A_{O} / R_{O}^{8}$, $B=B_{O} / R_{O}^{4}, h(t)=\exp \left[(6 / \sqrt{15}) t^{-1}((2 t+1) / \sqrt{15})\right]$, and then (10) and (11) could be

$$
\begin{aligned}
& \eta^{8}=\frac{A t^{6} h(t)}{\left[(1-t)^{4}\left(t^{2}+t+4\right)\right]} \\
& \zeta^{4}=\frac{B\left(t^{2}+t+4\right) h(t)}{\left[(1-t)^{2}\right]} \\
& \zeta_{r}=1-\frac{1}{\left(\lambda_{r}^{3} \lambda_{\theta}\right)} \\
& \zeta_{\theta}=1-\frac{1}{\left(\lambda_{\theta}^{3} \lambda_{r}\right)} \\
& \zeta_{Z}=1-\frac{1}{\left(\lambda_{r} \lambda_{\theta}\right)}
\end{aligned}
$$

\section{Mathematical Description of Rubber Cylinder under Compression}

3.1. Differential Equation Description of Rubber Cylinder. If the axial length is far greater than the thickness, in the axisymmetric problem, the axial direction of rubber cylinder can not be extended or shortened, so it can be simplified as axisymmetric plane strain problem, the calculation method of deformation mode, and main elongation are consistent with sponge cylinder. As rubber is superelastic material, its constitutive relation is defined by the partial derivative of the corresponding strain energy function $[20,21]$.

$$
s=\frac{2}{J} \operatorname{dev}\left[\left(\frac{\partial W}{\partial \bar{I}_{1}}+\bar{I}_{1} \frac{\partial W}{\partial \bar{I}_{2}}\right) \bar{B}-\frac{\partial W}{\partial \bar{I}_{2}} \bar{B} \cdot \bar{B}\right]
$$

where $s$ is the Cauchy stress deviation, $J$ is volume ratio before and after deformation, $\operatorname{dev}(\cdot)$ is tensor deviation operator, $W$ is the strain energy density converted by unit volume before deformation, $F$ is the deformation gradient tensor, then $B=F \cdot F^{T}, \bar{B}=J^{-2 / 3} \cdot B[22] ; I_{1}=\operatorname{tr}(B)$, $I_{2}=I_{1}^{2}-\operatorname{tr}\left(B \cdot B^{T}\right) ; \overline{I_{1}}=J^{-2 / 3} I_{1}, \overline{I_{2}}=J^{-4 / 3} I_{2} ; J=\operatorname{det}(F)$.
For incompressible sponge material, $J=1$. In terms of axisymmetric plane strain problem, (15) can be written as

$$
\begin{aligned}
& s=\frac{2}{J} \operatorname{dev}\left[\left(\frac{\partial W}{\partial \bar{I}_{1}}+\bar{I}_{1} \frac{\partial W}{\partial \bar{I}_{2}}\right) \bar{B}-\frac{\partial W}{\partial \bar{I}_{2}} \bar{B} \cdot \bar{B}\right] \\
& (i=r, \theta, z)
\end{aligned}
$$

It could be obtained from (15) as

$$
\begin{gathered}
\sigma_{\mathrm{r}}-\sigma_{\theta}=\left(\sigma_{\mathrm{r}}-p\right)-\left(\sigma_{\theta}-p\right)=s_{p}-s_{\theta} \\
=2\left[\left(\frac{\partial W}{\partial \bar{I}_{1}}+\bar{I}_{1} \frac{\partial W}{\partial \bar{I}_{2}}\right)\left(\frac{\lambda_{r}}{\lambda_{\theta}}-\frac{\lambda_{\theta}}{\lambda_{r}}\right)\right. \\
\left.-\frac{\partial W}{\partial \bar{I}_{2}}\left(\frac{\lambda_{r}^{2}}{\lambda_{\theta}^{2}}-\frac{\lambda_{\theta}^{2}}{\lambda_{r}^{2}}\right)\right]
\end{gathered}
$$

where $p$ is the hydrostatic pressure.

Equation (4) is the partial derivative of present coordinate of $r$; combining with (2), it could be written as the equation of initial coordinate $R$.

$$
\frac{d \sigma_{r}}{d R}+\frac{\lambda_{r}}{R \lambda_{\theta}}\left(\sigma_{r}-\sigma_{\theta}\right)=0
$$

The differential equation of axisymmetric plane strain could be obtained by submitting (16) into (17).

$$
\begin{gathered}
R \frac{d \sigma_{r}}{d R}+\frac{2 \lambda_{r}}{\lambda_{\theta}}\left[\left(\frac{\partial W}{\partial \bar{I}_{1}}+\bar{I}_{1} \frac{\partial W}{\partial \bar{I}_{2}}\right)\left(\frac{\lambda_{r}}{\lambda_{\theta}}-\frac{\lambda_{\theta}}{\lambda_{r}}\right)\right. \\
\left.-\frac{\partial W}{\partial \bar{I}_{2}}\left(\frac{\lambda_{r}^{2}}{\lambda_{\theta}^{2}}-\frac{\lambda_{\theta}^{2}}{\lambda_{r}^{2}}\right)\right]=0
\end{gathered}
$$

3.2. Differential Equation Analysis. In this paper, the threereduced polynomial constitutive model is used as the strain energy function of the rubber material. Because the volume of the rubber is incompressible, the strain energy function of the rubber is as follows $[23,24]$ :

$$
W=C_{10}\left(I_{1}-3\right)+C_{20}\left(I_{1}-3\right)^{2}+C_{30}\left(I_{1}-3\right)^{3}
$$

In terms of axisymmetric plane strain, $\lambda_{z}=1$ and (19) is submitted into (18) to obtain

$$
\begin{aligned}
R \frac{d \sigma_{r}}{d R}+2\left[C_{10}+C_{20}\left(I_{1}-3\right)+C_{30}\left(I_{1}-3\right)^{2}\right] \\
\cdot\left[\left(\frac{\lambda_{r}}{\lambda_{\theta}}\right)^{2}-1\right]=0
\end{aligned}
$$

As the rubber is incompressible and combining with (2),

$$
\frac{d r}{d R}=\frac{R}{r}
$$

The deformation function of rubber cylinder could be obtained by integral.

$$
r^{2}=R^{2}+C_{0}
$$


where $C_{0}$ is integral constant and is determined by the boundary condition.

Equations (21) and (22) are substituted into (20):

$$
\begin{aligned}
\frac{d \sigma_{r}}{d R}= & -\frac{6 C_{30} R^{7}}{\left(R^{2}+C_{0}\right)^{4}}-\frac{4\left(C_{20}-6 C_{30}\right) R^{5}}{\left(R^{2}+C_{0}\right)^{3}} \\
& -\frac{2\left(C_{10}-4 C_{20}+15 C_{30}\right) R^{3}}{\left(R^{2}+C_{0}\right)^{2}} \\
& +\frac{6 C_{30}\left(R^{2}+C_{0}\right)^{2}}{R^{5}} \\
& +\frac{4\left(C_{20}-6 C_{30}\right)\left(R^{2}+C_{0}\right)}{R^{3}} \\
& +\frac{2\left(C_{10}-4 C_{20}+15 C_{30}\right)}{R}
\end{aligned}
$$

By integrating (23), the radial stress of rubber cylinder is as follows:

$$
\begin{aligned}
\sigma_{r}= & -\frac{C_{30} C_{0}^{3}}{\left(R^{2}+C_{0}\right)^{3}}+\frac{\left(2 C_{20}-3 C_{30}\right) C_{0}^{2}}{2\left(R^{2}+C_{0}\right)^{2}}-\frac{C_{10} C_{0}}{\left(R^{2}+C_{0}\right)} \\
& -\frac{3 C_{30} C_{0}^{2}}{2 R^{4}}-\frac{2\left(C_{20}-3 C_{30}\right) C_{0}}{R^{2}} \\
& -\left(C_{10}-2 C_{20}+6 C_{30}\right) \ln \left(1+\frac{C_{0}}{R^{2}}\right)+D_{0}
\end{aligned}
$$

where $D_{0}$ is integral constant and is determined by the boundary condition.

Combining (16) with (24), the tangential stress of the rubber cylinder is as follows:

$$
\begin{aligned}
\sigma_{\theta}= & \sigma_{r}+6 C_{30}\left(\frac{\left(R^{2}+C_{0}\right)^{5}}{R^{6}}+\frac{R^{6}}{\left(R^{2}+C_{0}\right)^{3}}\right) \\
& +4\left(C_{20}-6 C_{30}\right)\left(\frac{\left(R^{2}+C_{0}\right)^{2}}{R^{4}}-\frac{R^{4}}{\left(R^{2}+C_{0}\right)^{2}}\right) \\
& +2\left(C_{10}-4 C_{20}+15 C_{30}\right) \\
& \cdot\left(\frac{\left(R^{2}+C_{0}\right)}{R^{2}}-\frac{R^{2}}{\left(R^{2}+C_{0}\right)}\right)
\end{aligned}
$$

3.3. Dimensionless of Deformation and Stress. Setting $\eta=$ $R / R_{O}$, which represents the dimensionless radius before deformation, $\xi=r / R$ represents the dimensionless radius after deformation, $\xi_{i}=\sigma_{i} / \mu$ represents the dimensionless stress, $C_{1}=C_{10} / \mu, C_{2}=C_{20} / \mu, C_{3}=C_{30} / \mu$, and $D=D_{0} / \mu$, where $\mu$ is the parameter of the Baltz-Ko constitutive model of

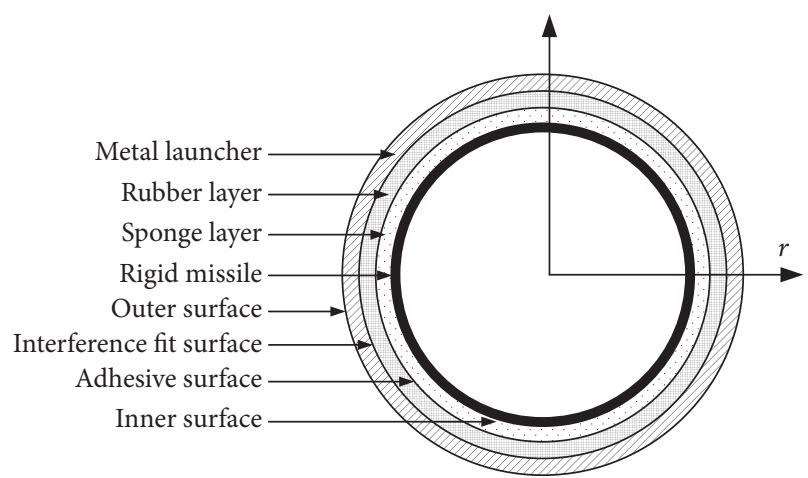

FIGURE 2: Schematic diagram of interference fit between adapters and metal launchers.

the sponge. Then the corresponding deformation mode and stress can be written as

$$
\begin{aligned}
\xi^{2}= & \eta^{2}+C \\
\xi_{r}= & -\frac{C_{3} C^{3}}{\left(\eta^{2}+C\right)^{3}}+\frac{\left(2 C_{2}-3 C_{3}\right) C^{2}}{2\left(\eta^{2}+C\right)^{2}}-\frac{C_{1} C}{\eta^{2}+C} \\
& -\frac{3 C_{3} C^{2}}{2 \eta^{4}}-\frac{2\left(C_{2}-3 C_{3}\right) C}{\eta^{2}} \\
& -\left(C_{1}-2 C_{2}+6 C_{3}\right) \ln \left(1+\frac{C}{\eta^{2}}\right)+D \\
\xi_{\theta}= & \xi_{r}+6 C_{3}\left(\frac{\left(\eta^{2}+C\right)^{3}}{\eta^{6}}-\frac{\eta^{6}}{\left(\eta^{2}+C\right)^{3}}\right) \\
& +4\left(C_{2}-6 C_{3}\right)\left(\frac{\left(\eta^{2}+C\right)^{2}}{\eta^{4}}-\frac{\eta^{4}}{\left(\eta^{2}+C\right)^{2}}\right) \\
& -2\left(C_{1}-4 C_{2}+15 C_{3}\right)\left(\frac{\eta^{2}+C}{\eta^{2}}-\frac{\eta^{2}}{\eta^{2}+C}\right)
\end{aligned}
$$

\section{Analysis of Interference Fit between Adapters and Launchers}

4.1. Geometric Description. Schematic diagram of interference fit between adapters and metal launchers is shown in Figure 2. The materials from inside to the outside are sponges, incompressible rubber, and isotropic metals and are, respectively, assigned as material 1 , material 2 , and material 3 . The subscript numbers 1,2 , and 3 in variable correspond to material 1 , material 2 , and material 3 . The variable with subscript I has relationships with inner surface of adapter, which with subscript $M$ has relationships with adhesive surface; the variable related to outer surface of launchers is marked with subscript $\mathrm{O}$.

The outer radius of launcher before deformation is $\eta_{3 O}=$ $\eta_{O}=1$ and the inner radius is $\eta_{3 I}$. Similarly, the adapter's outer radius is $\eta_{2 O}$ before deformation, inner diameter is $\eta_{1 I}=\eta_{I}$, and the adhesive surface radius is $\eta_{M}$. The 
interference fit is adopted between rubber's outer diameter and launcher's inner diameter, and the precompression is $\delta$. After the installation of adapter into the launcher, the radius after deformation is, respectively, as follows, for the launchers, the outer radius is $\xi_{3 O}=\xi_{O}$ and the inner is $\xi_{3 I}$. For the adapter, the outer radius is $\xi_{2 O}$, the adhesive radius is $\xi_{M}$ and the inner radius is $\xi_{1 I}=\xi_{I}$. Nevertheless, $\xi_{r 3 I}$ and $\xi_{O}$ are all unknown and the outer surface pressure of launcher is 0 .

4.2. Axisymmetric Plane Strain Solution for Launcher. Considering that the mechanical properties of the launcher are isotropic and without volume force, the boundary value problem is attributed to [25-28]

$$
\begin{aligned}
\nabla^{4} F & =\left(\frac{d^{2}}{d R^{2}}+\frac{1}{R} \frac{d}{d R}\right)^{2} F=0 \\
\left.\sigma_{r}\right|_{R=R_{O}} & =-\sigma_{r O}, \\
\left.\tau_{r 9}\right|_{R=R_{O}} & =0, \\
\left.\sigma_{r}\right|_{R=R_{I}} & =-\sigma_{r I}, \\
\left.\tau_{r \vartheta}\right|_{R=R_{I}} & =0
\end{aligned}
$$

where $F$ is the stress function and is the function of $r$ and $\theta$ under polar coordinates. For the axisymmetric issue, $F$ is the function of $r$ and has nothing with $\theta$.

If the stress function $F$ is known, then its stress could be expressed as [25-28]

$$
\begin{aligned}
\sigma_{r} & =\frac{1}{R} \frac{\partial F}{\partial R}+\frac{1}{R^{2}} \frac{\partial^{2} F}{\partial R^{2}} \\
\sigma_{\theta} & =\frac{\partial^{2} F}{\partial R^{2}} \\
\tau_{r \theta} & =\tau_{\theta r}=\frac{1}{R^{2}} \frac{\partial F}{\partial \theta}-\frac{1}{R} \frac{\partial^{2} F}{\partial R \partial \theta}=-\frac{\partial}{\partial R}\left(\frac{1}{R} \frac{\partial F}{\partial \theta}\right)
\end{aligned}
$$

Considering the operator of $d^{2} / d R^{2}+(1 / R)(d / d R)=$ $(1 / R)(d / d R) R(d / d R)$, then (29) could be written as

$$
\nabla^{4} F=\frac{1}{R} \frac{d}{d R} R \frac{d}{d R} \frac{1}{R} \frac{d}{d R} R \frac{d F}{d R}=0
$$

The integrating on (32) to obtain

$$
F(R)=J_{0} R^{2} \ln R+K_{0} \ln R+M_{0} R^{2}+N_{0}
$$

Then the corresponding stress component is as follows:

$$
\begin{aligned}
& \sigma_{r}=J_{0}(1+2 \ln R)+\frac{K_{0}}{R^{2}}+2 M_{0}, \\
& \sigma_{\theta}=J_{0}(3+2 \ln R)-\frac{K_{0}}{R^{2}}+2 M_{0}, \\
& \tau_{r \theta}=\tau_{\theta r}=0
\end{aligned}
$$

where $J_{0}, K_{0}, M_{0}$, and $N_{0}$ are the integral constant, which are determined by the boundary condition and single value condition of displacement.
Considering the axial symmetry, the small deformation geometric function is as follows:

$$
\begin{gathered}
\varepsilon_{r}=\frac{\partial u_{r}}{\partial R}, \\
\varepsilon_{\theta}=\frac{u_{r}}{R}+\frac{1}{R} \frac{\partial u_{\theta}}{\partial \theta}, \\
\gamma_{r \theta=} \gamma_{\theta r=} \frac{1}{R} \frac{\partial u_{r}}{\partial \theta}+\frac{\partial u_{\theta}}{\partial R}-\frac{u_{\theta}}{R}
\end{gathered}
$$

And the constitutive function of plane stress is as follows:

$$
\begin{aligned}
\varepsilon_{r} & =\frac{1-\nu^{2}}{E}\left(\sigma_{r}-\frac{\nu}{1-\nu} \sigma_{\theta}\right), \\
\varepsilon_{\theta} & =\frac{1-\nu^{2}}{E}\left(\sigma_{\theta}-\frac{\nu}{1-\nu} \sigma_{r}\right), \\
\gamma_{r \theta} & =\frac{1}{G} \tau_{r \theta}
\end{aligned}
$$

The strain component could be calculated by constitutive (35b) and then be submitted into boundary value (30). Due to the single value condition of the displacement, the N0 $=0$ and the integral constants can be obtained.

$$
\begin{aligned}
L_{0} & =\frac{R_{I}^{2} R_{O}^{2}\left(\sigma_{r 1}-\sigma_{r O}\right)}{R_{O}^{2}-R_{I}^{2}} \\
2 M_{0} & =\frac{R_{O}^{2} \sigma_{r O}-R_{I}^{2} \sigma_{r 1}}{R_{O}^{2}-R_{I}^{2}} \\
\sigma_{r} & =\frac{R_{I}^{2} R_{O}^{2}\left(\sigma_{r 1}-\sigma_{r O}\right)}{R_{O}^{2}-R_{I}^{2}} \cdot \frac{1}{R^{2}} \frac{R_{O}^{2} \sigma_{r O}-R_{I}^{2} \sigma_{r 1}}{R_{O}^{2}-R_{I}^{2}} \\
\sigma_{\theta} & =-\frac{R_{I}^{2} R_{O}^{2}\left(\sigma_{r 1}-\sigma_{r O}\right)}{R_{O}^{2}-R_{I}^{2}} \cdot \frac{1}{R^{2}} \frac{R_{O}^{2} \sigma_{r O}-R_{I}^{2} \sigma_{r 1}}{R_{O}^{2}-R_{I}^{2}} \\
\sigma_{z} & =v\left(\sigma_{r}+\sigma_{\theta}\right) \\
u_{r} & =\frac{1+\nu}{E}\left[\frac{R_{I}^{2} R_{O}^{2}\left(\sigma_{r 1}-\sigma_{r O}\right)}{R_{O}^{2}-R_{I}^{2}} \cdot \frac{1}{R}\right. \\
& \left.+(1-2 \nu) \frac{R_{O}^{2} \sigma_{r O}-R_{I}^{2} \sigma_{r 1}}{R_{O}^{2}-R_{I}^{2}} \cdot R\right], \\
u_{\theta} & =0
\end{aligned}
$$

Assuming that $\xi_{i}=\sigma_{i} / \mu, \psi=\xi-\eta$, and $E^{\prime}=E / \mu$, the dimensionless is carried out on (36) and (37) to obtain

$$
\begin{aligned}
& \zeta_{r}=\frac{\eta_{I}^{2} \eta_{O}^{2}\left(\zeta_{r 1}-\zeta_{r O}\right)}{\eta_{O}^{2}-\eta_{I}^{2}} \cdot \frac{1}{\eta^{2}}+\frac{\eta_{O}^{2} \zeta_{r O}-\eta_{I}^{2} \eta_{r 1}}{\eta_{O}^{2}-\eta_{I}^{2}} \\
& \zeta_{\theta}=-\frac{\eta_{I}^{2} \eta_{O}^{2}\left(\zeta_{r 1}-\zeta_{r O}\right)}{\eta_{O}^{2}-\eta_{I}^{2}} \cdot \frac{1}{\eta^{2}}+\frac{\eta_{O}^{2} \zeta_{r O}-\eta_{I}^{2} \eta_{r 1}}{\eta_{O}^{2}-\eta_{I}^{2}} \\
& \zeta_{z}=v\left(\zeta_{r}+\zeta_{\theta}\right)
\end{aligned}
$$




$$
\begin{aligned}
\psi_{r} & =\frac{1+\nu}{E^{\prime}}\left[\frac{\eta_{I}^{2} \eta_{O}^{2}\left(\zeta_{r O}-\zeta_{r I}\right)}{\eta_{O}^{2}-\eta_{I}^{2}} \cdot \frac{1}{\eta}\right. \\
& \left.+(1+2 \nu) \frac{\eta_{O}^{2} \zeta_{r O}-\eta_{I}^{2} \eta_{r 1}}{\eta_{O}^{2}-\eta_{I}^{2}} \cdot \eta\right], \\
u_{\theta} & =0
\end{aligned}
$$

4.3. Equation Solving of Interference Fit Problem. Before the adapter installing into the launcher, the sizes of adapter are, respectively, $\eta_{2 O}, \eta_{M}, \eta_{1 I}=\eta_{I}$. The size of launcher before deformation is $\eta_{3 O}=\eta_{O}=1$. As $\eta_{3 I}$ is known, $\xi_{3 I}=\xi_{2 O}$ is obtained after the adapter installing into launcher.

From (39), the relation between the inner surface pressure and the radial displacement of the launcher could be obtained.

$$
\psi_{r 3 I}=-\frac{1+\nu}{E^{\prime}}\left[\frac{\eta_{3 O}^{2}+(1-2 \nu) \eta_{3 I}^{2}}{\eta_{3 O}^{2}-\eta_{3 I}^{2}}\right] \eta_{3 I} \zeta_{3 I}
$$

The inner radius of launcher after deformation is

$$
\begin{aligned}
\xi_{3 I} & =\eta_{3 I}+\psi_{r 3 I} \\
& =\eta_{3 I}-\frac{1+\nu}{E^{\prime}}\left[\frac{\eta_{3 O}^{2}+(1-2 \nu) \eta_{3 I}^{2}}{\eta_{3 O}^{2}-\eta_{3 I}^{2}}\right] \eta_{3 I} \zeta_{3 I}
\end{aligned}
$$

Equation (41) could be written as

$$
\xi_{3 I}=a+b \zeta_{r 3 I}
$$

where $a=\eta_{3 I}$ and $b=-\left((1+v) / E^{\prime}\right)\left[\left(\eta_{3 O}^{2}+(1-2 v) \eta_{3 I}^{2}\right) /\left(\eta_{3 O}^{2}-\right.\right.$ $\left.\left.\eta_{3 I}^{2}\right)\right] \eta_{3 I}$.

According (22), the radius expression of adapter and adhesive surface after deformation is

$$
\begin{aligned}
& \xi_{3 I}^{2}=\xi_{2 O}^{2}=\eta_{2 O}^{2}+C \\
& \xi_{M}^{2}=\eta_{M}^{2}+C
\end{aligned}
$$

After the deformation of adapter, the radial pressure of the outer diameter of the rubber layer and the inner diameter of the launcher must be equal. According to (24), the radial pressure expression of the inner layer of the launcher can be written out.

$$
\begin{aligned}
\xi_{r 3 I}= & -\frac{C_{3} C^{3}}{\left(\eta_{2 O}^{2}+C\right)^{3}}+\frac{\left(2 C_{2}-3 C_{3}\right) C^{2}}{2\left(\eta_{2 O}^{2}+C\right)^{2}}-\frac{C_{1} C}{\eta_{2 O}^{2}+C} \\
& -\frac{3 C_{3} C^{2}}{2 \eta_{2 O}^{4}}-\frac{2\left(C_{2}-3 C_{3}\right) C}{\eta_{2 O}^{2}} \\
& -\left(C_{1}-2 C_{2}+6 C_{3}\right) \ln \left(1+\frac{C}{\eta_{2 O}^{2}}\right)+D
\end{aligned}
$$

Equation (42) is submitted into (45), then

$$
\begin{aligned}
\frac{\sqrt{\eta_{2 O}^{2}+C}-a}{b}= & -\frac{C_{3} C^{3}}{\left(\eta_{2 O}^{2}+C\right)^{3}}+\frac{\left(2 C_{2}-3 C_{3}\right) C^{2}}{2\left(\eta_{2 O}^{2}+C\right)^{2}} \\
& -\frac{C_{1} C}{\eta_{2 O}^{2}+C}-\frac{3 C_{3} C^{2}}{2 \eta_{2 O}^{4}} \\
& -\frac{2\left(C_{2}-3 C_{3}\right) C}{\eta_{2 O}^{2}} \\
& -\left(C_{1}-2 C_{2}+6 C_{3}\right) \ln \left(1+\frac{C}{\eta_{2 O}^{2}}\right) \\
& +D
\end{aligned}
$$

The following two equations can be obtained by bringing the initial internal and external radius of the material 1 in the adapter into (12).

$$
\begin{aligned}
\eta_{M}^{8} & =\frac{A t_{M}^{6} h\left(t_{M}\right)}{\left(1-t_{M}\right)^{4}\left(t_{M}^{2}+t_{M}+4\right)} \\
\eta_{I}^{8} & =\frac{A t_{I}^{6} h\left(t_{I}\right)}{\left(1-t_{I}\right)^{4}\left(t_{I}^{2}+t_{I}+4\right)}
\end{aligned}
$$

Considering the radius of the adapter's adhesive surface after deformation, (44) is brought into (13):

$$
\left(\eta_{M}^{2}+C\right)^{2}=\frac{B\left(t_{M}^{2}+t_{M}+4\right) h\left(t_{M}\right)}{\left(1-t_{M}\right)^{2}}
$$

Due to the assumption that the body is rigid, the inner diameter of the adapter will not deform, so the inner diameter of the adapter is known and can be obtained:

$$
\xi_{I}^{4}=\frac{B\left(t_{I}^{2}+t_{I}+4\right) h\left(t_{I}\right)}{\left(1-t_{I}\right)^{2}}
$$

As the spongy and rubber materials are in a continuous radial stress in the adhesive surface, a similar equation (46) can be written out.

$$
\begin{aligned}
1-\frac{1}{\lambda_{r M}^{3} \lambda_{\theta M}}= & -\frac{C_{3} C^{3}}{\left(\eta_{M}^{2}+C\right)^{3}}+\frac{\left(2 C_{2}-3 C_{3}\right) C^{2}}{2\left(\eta_{M}^{2}+C\right)^{2}} \\
& -\frac{C_{1} C}{\eta_{M}^{2}+C}-\frac{3 C_{3} C^{2}}{2 \eta_{M}^{4}} \\
& -\frac{2\left(C_{2}-3 C_{3}\right) C}{\eta_{M}^{2}} \\
& -\left(C_{1}-2 C_{2}+6 C_{3}\right) \ln \left(1+\frac{C}{\eta_{M}^{2}}\right) \\
& +D
\end{aligned}
$$


According to $t=\lambda_{r} / \lambda_{\theta}=R r^{\prime} / r, \lambda_{r M}=t_{M} \lambda_{\theta M}$ could be obtained at the cohesive surface, where $\lambda_{\theta M}=\xi_{M} / \eta_{M}$. $\lambda_{r M}=t_{M} \sqrt{1+C / \eta_{M}^{2}}$ could be obtained based on (44); thus (51) could be written as (51).

$$
\begin{aligned}
1- & \frac{1}{t_{M}^{3}\left(1+C / \eta_{M}^{2}\right)} \\
= & -\frac{C_{3} C^{3}}{\left(\eta_{M}^{2}+C\right)^{3}}+\frac{\left(2 C_{2}-3 C_{3}\right) C^{2}}{2\left(\eta_{M}^{2}+C\right)^{2}}-\frac{C_{1} C}{\eta_{M}^{2}+C} \\
& -\frac{3 C_{3} C^{2}}{2 \eta_{M}^{4}}-\frac{2\left(C_{2}-3 C_{3}\right) C}{\eta_{M}^{2}} \\
& -\left(C_{1}-2 C_{2}+6 C_{3}\right) \ln \left(1+\frac{C}{\eta_{M}^{2}}\right)+D
\end{aligned}
$$

Equations (46), (47), (48), (49), (50), and (51) are transformed properly, and they are rewritten into equations forms.

$$
\begin{aligned}
& F(X) \\
& =\left\{f_{1}(X) f_{2}(X) f_{3}(X) f_{4}(X) f_{5}(X) f_{6}(X)\right\}^{T} \\
& =0
\end{aligned}
$$

where $X=\left\{\begin{array}{llllll}A & B & C & D & t_{M} & t_{I}\end{array}\right\}^{T}$.

$$
\begin{aligned}
f_{1}(X)= & \frac{\sqrt{\eta_{2 O}^{2}+C}-a}{b}+\frac{C_{3} C^{3}}{\left(\eta_{2 O}^{2}+C\right)^{3}} \\
& -\frac{\left(2 C_{2}-3 C_{3}\right) C^{2}}{2\left(\eta_{2 O}^{2}+C\right)^{2}}+\frac{C_{1} C}{\eta_{2 O}^{2}+C}+\frac{3 C_{3} C^{2}}{2 \eta_{2 O}^{4}} \\
& +\frac{2\left(C_{2}-3 C_{3}\right) C}{\eta_{2 O}^{2}} \\
& +\left(C_{1}-2 C_{2}+6 C_{3}\right) \ln \left(1+\frac{C}{\eta_{2 O}^{2}}\right)-D \\
f_{2}(X)= & 1-\frac{\eta_{M}^{4}}{t_{M}^{3}\left(\eta_{M}^{2}+C\right)}+\frac{C_{3} C^{3}}{\left(\eta_{M}^{2}+C\right)^{3}} \\
& -\frac{\left(2 C_{2}-3 C_{3}\right) C^{2}}{2\left(\eta_{M}^{2}+C\right)^{2}}+\frac{C_{1} C}{\eta_{M}^{2}+C}+\frac{3 C_{3} C^{2}}{2 \eta_{M}^{4}} \\
& +\frac{2\left(C_{2}-3 C_{3}\right) C}{\eta_{M}^{2}} \\
& +\left(C_{1}-2 C_{2}+6 C_{3}\right) \ln \left(1+\frac{C}{\eta_{M}^{2}}\right)-D \\
f_{3}(X)= & A t_{M}^{6} h\left(t_{M}\right)-\eta_{M}^{8}\left(1-t_{M}\right)^{4}\left(t_{M}^{2}+t_{M}+4\right)
\end{aligned}
$$

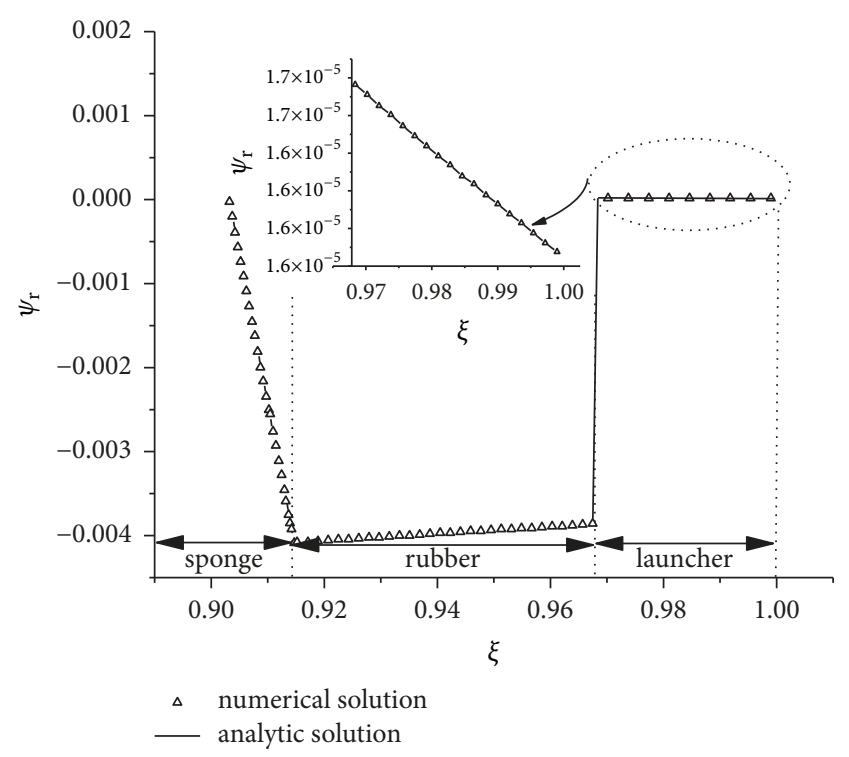

Figure 3: Radial displacement curve.

$$
\begin{aligned}
f_{4}(X)= & A t_{I}^{6} h\left(t_{I}\right)-\eta_{I}^{8}\left(1-t_{I}\right)^{4}\left(t_{I}^{2}+t_{I}+4\right) \\
f_{5}(X)= & B\left(t_{M}^{2}+t_{M}+4\right) h\left(t_{M}\right) \\
& -\left(\eta_{M}^{2}+C\right)\left(1-t_{M}\right)^{2} \\
f_{6}(X)= & B\left(t_{I}^{2}+t_{I}+4\right) h\left(t_{I}\right)-\xi_{I}^{4}\left(1-t_{I}\right)^{2}
\end{aligned}
$$

The nonlinear equation group (53) is calculated by using Newton-Raphson method $[29,30]$, and the $A, B, C, D, t_{\mathrm{M}}$, and $t_{\mathrm{I}}$ could be obtained, and then the deformation and stress of the interference fit for the adapter and the launcher could be obtained.

4.4. Example Verification and Analysis. The precompression $\delta$ of the adapter is 0.005161 in a certain missile launching system, the dimensionless sizes are $\eta_{3 I}=0.967742, \eta_{2 O}=$ $0.971613, \eta_{M}=0.918710$, and $\eta_{I}=\xi_{I}=0.903226$. The material parameters of adapter and launcher are $C_{1}=185.0$, $C_{2}=6.5660, C_{3}=0.03488, E^{\prime}=2.954595 \times 10^{6}$, and $v=$ 0.340 . The finite element software ANSYS is adopted and the radial displacement, radial stress, and tangential stress curves of adapter and launcher are obtained, as shown in Figure 5.

From Figures 3-5, the displacement, radial stress, and tangential stress of analytical solution are consistent with that of the finite element. As a result, the analytic formula of interference fit of deformation and stress is deduced correctly in this paper, which is applicable to the sponge cylinder, the rubber cylinder, the adapter, and the launcher.

The maximum absolute value of adapter's displacement is 0.0040772 in Figure 3, which appears on the adhesive surface and is larger than the precompression of adapter. As indicated, the rubber layer will move onward as a whole when the adapter is subjected to pressure. The maximum absolute value of adapter's outer surface displacement is 


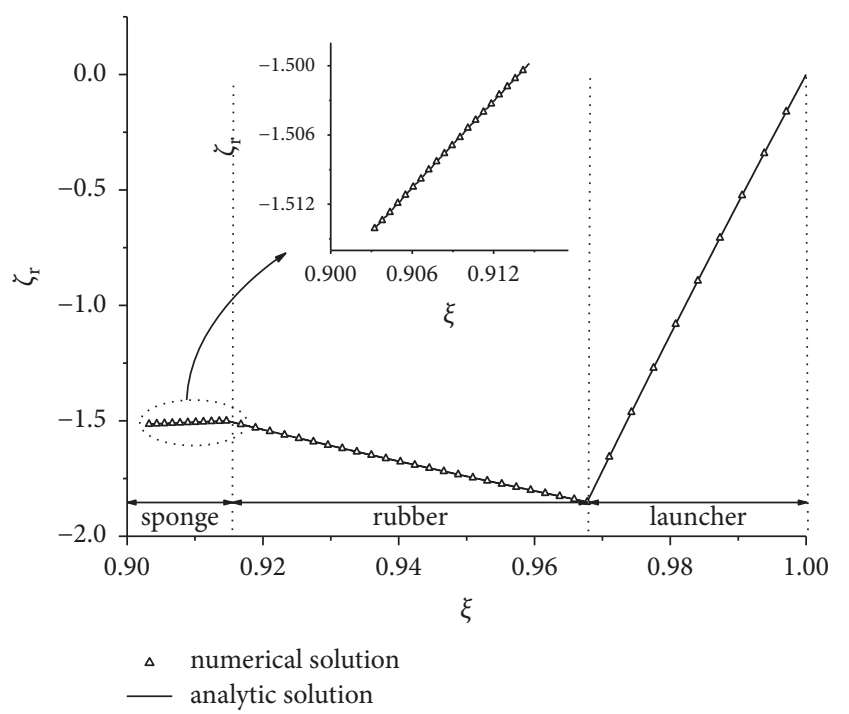

FIgURE 4: Radial stress curve.

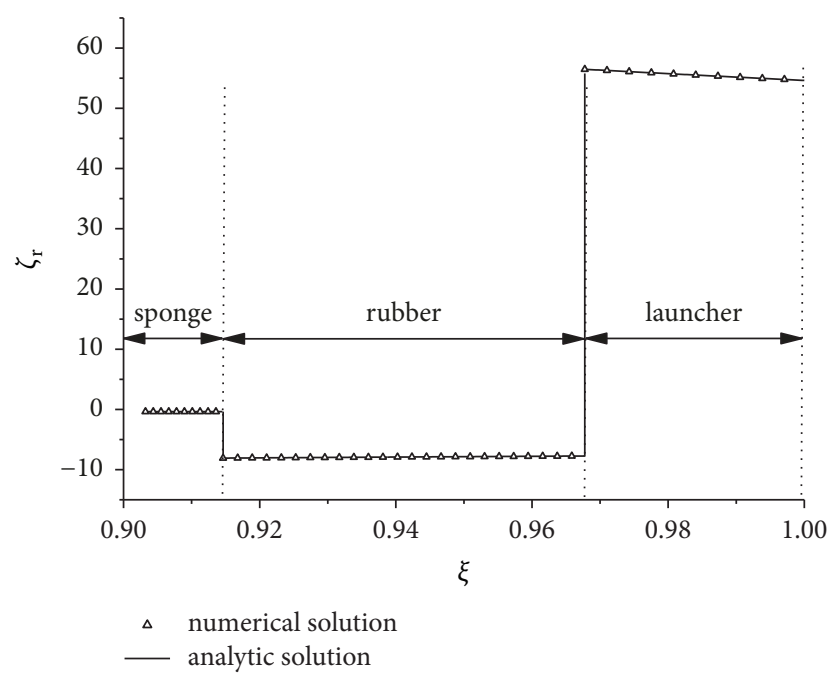

FIgURE 5: Tangential stress curve.

0.0038593 with an inward movement. After the installation of adapter into the launcher, every point in the launcher moves outward; the maximum displacement of launcher appears on the interference fit surface with the value of $1.663775 \times 10^{-5}$, which is far smaller than that of adapter. The outer surface displacement of launcher is $1.66361 \times 10^{-5}$.

It could be concluded in Figure 4 that the absolute value of sponge's radial stress in the adapter turns small from the inside to the outside, while that of rubber layer turns large. From inside to outside, the absolute value of radial stress in launcher reduced to 0 , and the three materials are all subjected to compression. The absolute value of the radial stress of the inner adapter is 1.5141, that on the adapter surface is 1.4999 , and that on the interference fit between the adapter and the launcher is 1.8462 .

In view of Figure 5, the absolute value of tangential stress of inner sponge layer in the adapter is 0.36058 , which increase along the radial direction, and that of outer sponge

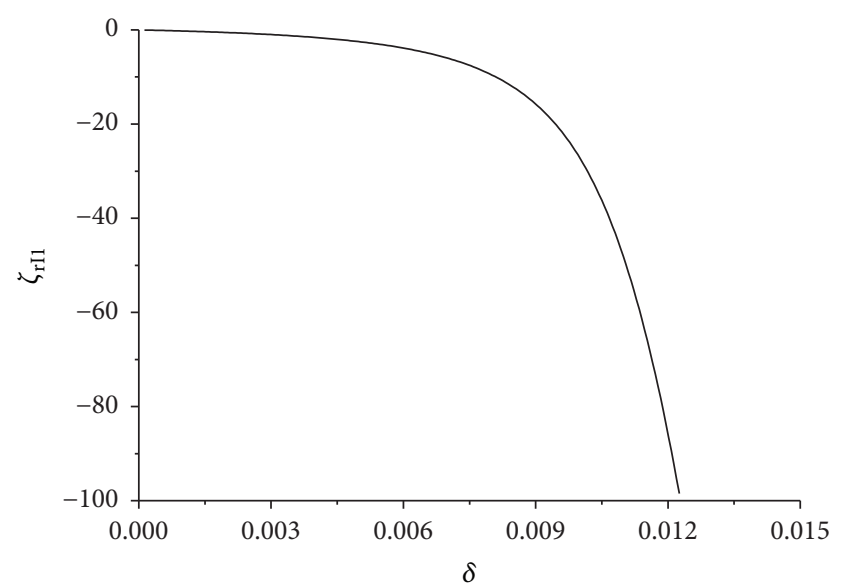

FIgURE 6: Effects to the $\zeta_{\text {rII }}$ of adapter.

layer reaches 0.37337 . For the absolute value of tangential stress in the rubber layer, it is 0.36058 in the inner surface and decreases radially and then reduces to 7.7368 on the outer surface. The tangential stress for the whole adapter is compressive stress while that of launcher is tensile stress. The tensile stress is 56.481 in the inner surface of launcher and decrease radically, which is 54.664 in the outer layer.

\section{Analysis on the Deformation and Stress Effects of Adapters}

5.1. Effect of Precompression on the Deformation and Stress. Figures 6-13 are effect curves of precompression $\delta$, respectively, to radial stress of inner sponge $\left(\zeta_{\mathrm{rI} 1}\right)$, to the tangential stress of inner sponge $\left(\zeta_{\theta \mathrm{I} 1}\right)$ and outer surface $\left(\zeta_{\theta \mathrm{M} 1}\right)$, the radial displacement of outer sponge $\left(\psi_{\mathrm{rM}}\right)$, the radial stress of outer sponge $\left(\zeta_{\mathrm{rM}}\right)$, the tangential stress of inner rubber $\left(\zeta_{\theta \mathrm{M} 2}\right)$ and outer surface $\left(\zeta_{\theta \mathrm{O} 2}\right)$, the tangential stress of the outer rubber surface $\left(\zeta_{\mathrm{rO} 2}\right)$, the radial displacement of inner launcher $\left(\psi_{\mathrm{r} 33}\right)$ and the outer radial displacement $\left(\psi_{\mathrm{rO} 3}\right)$, the tangential stress of the inner launcher $\left(\zeta_{\theta \mathrm{I} 3}\right)$, and tangential stress of outer surface $\left(\zeta_{\theta \mathrm{O} 3}\right)$.

From Figures 6-13, the effects of $\delta$ to $\zeta_{\mathrm{rI} 1}, \zeta_{\mathrm{rM}}, \zeta_{\theta \mathrm{M} 2}, \zeta_{\theta \mathrm{O} 2}$, $\zeta_{\mathrm{rO} 2}, \psi_{\mathrm{rI} 3}, \psi_{\mathrm{rO} 3}, \zeta_{\theta \mathrm{I} 3}$, and $\zeta_{\theta \mathrm{O} 3}$ are divided onto three stages:

(1) When $0 \leq \delta \leq 0.0075$ at the first stage, $\zeta_{\mathrm{rI} 1}, \zeta_{\mathrm{rM}}$, $\zeta_{\mathrm{\theta M} 2}, \zeta_{\theta \mathrm{O} 2}, \zeta_{\mathrm{rO} 2}, \psi_{\mathrm{rI} 3}, \psi_{\mathrm{rO} 3}, \zeta_{\theta \mathrm{I} 3}$, and $\zeta_{\theta \mathrm{O} 3}$ is approximately proportion to the precompression $\delta$. The absolute value of proportional coefficient is small and the precompression $\delta$ varies largely, while the corresponding stress and displacement change little.

(2) When $0.0075 \leq \delta \leq 0.0105$ at the second stage, with the change of precompression $\delta$, the change rates of $\zeta_{\mathrm{rI} 1}, \zeta_{\mathrm{rM}}$, $\zeta_{\theta \mathrm{M} 2}, \zeta_{\theta \mathrm{O} 2}, \zeta_{\mathrm{rO} 2}, \psi_{\mathrm{rI} 3}, \psi_{\mathrm{rO} 3}, \zeta_{\theta \mathrm{I} 3}$, and $\zeta_{\theta \mathrm{O} 3}$ are getting faster and faster.

(3) When $\delta \geq 0.0105$ at the third stage, the precompression $\delta$ changes little while the corresponding stress and displacement change largely.

5.2. Effect of Thickness Ratio of the Sponge Layer on the Deformation and Stress. The thickness ratio of sponge layer is 


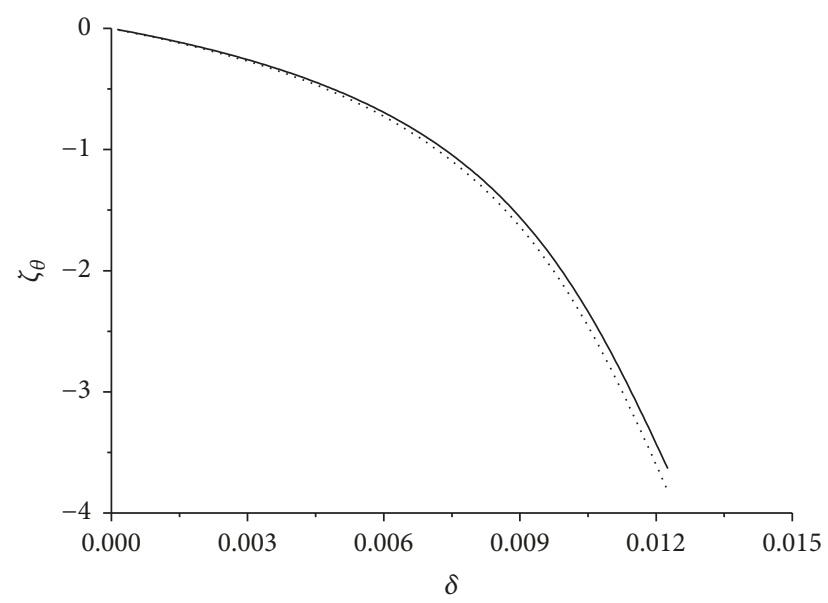

$-\zeta_{\theta 11}$

FIGURE 7: Effects to the $\zeta_{\theta \mathrm{I} 1}$ and $\zeta_{\theta \mathrm{M} 1}$ of adapter.

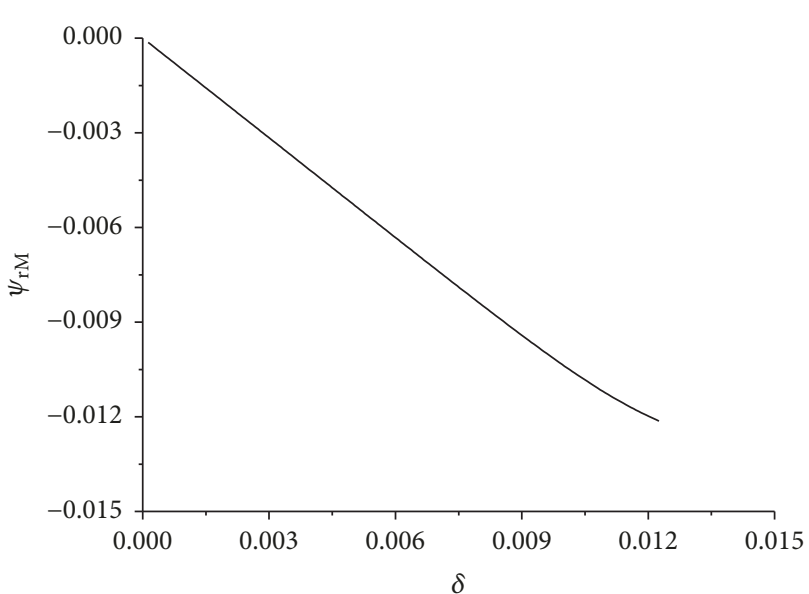

FIGURE 8: Effects to the $\psi_{\mathrm{rM}}$ of adapter

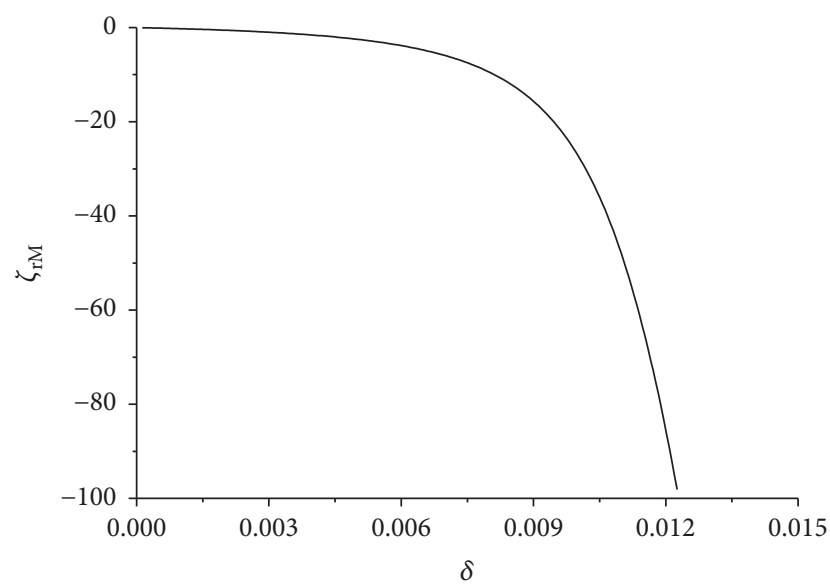

Figure 9: Effects to the $\zeta_{\mathrm{rM}}$ of adapter.

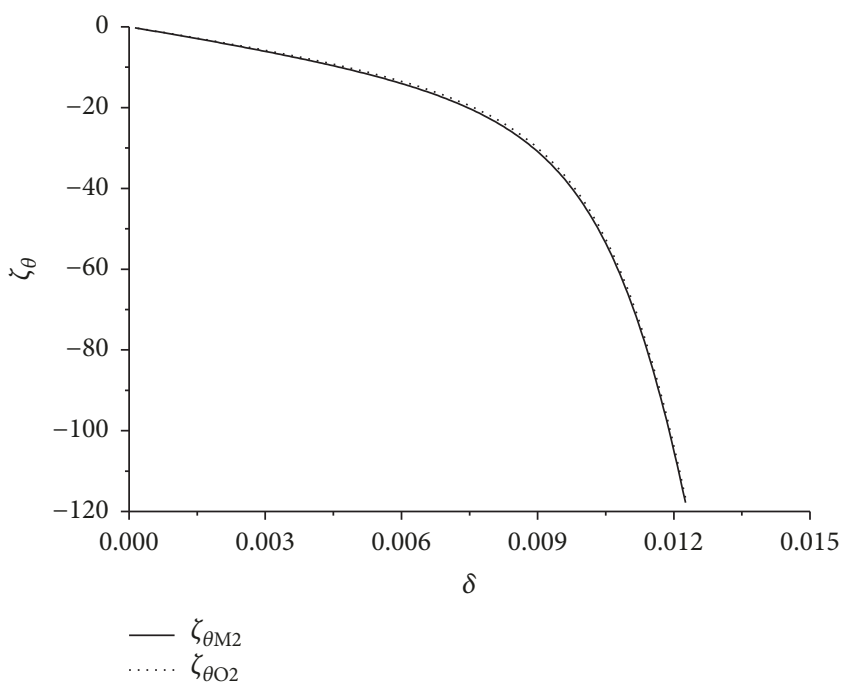

FIGURE 10: Effects to the $\zeta_{\theta \mathrm{M} 2}$ and $\zeta_{\theta \mathrm{O} 2}$ of adapter.

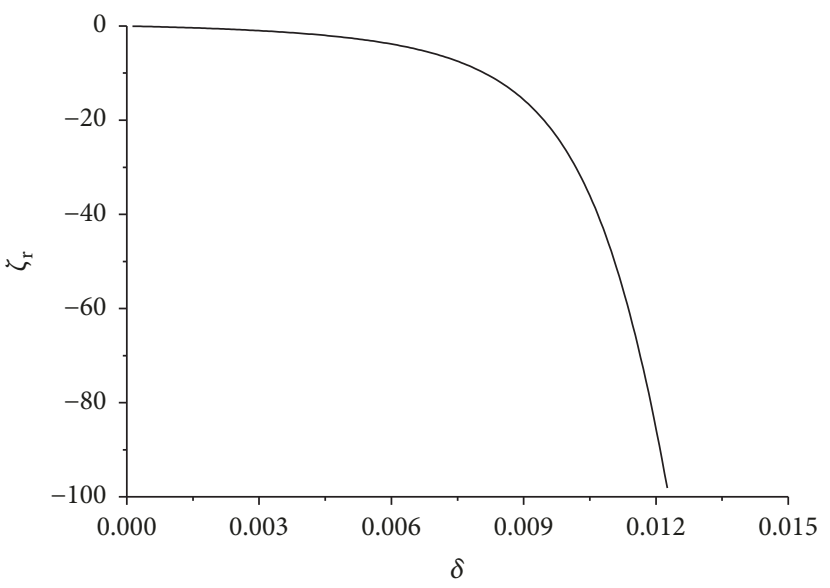

FIGURE 11: Effects of $\delta$ to the $\zeta_{\mathrm{rO} 2}$ of adapter.

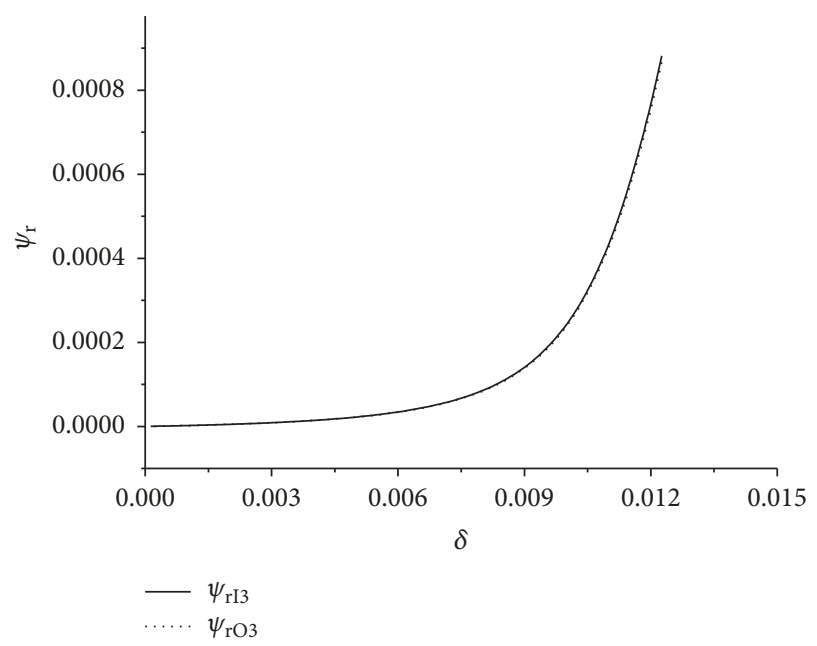

FIGURE 12: Effects to the $\psi_{\mathrm{rI} 3}$ and $\psi_{\mathrm{rO} 3}$ of adapter. 


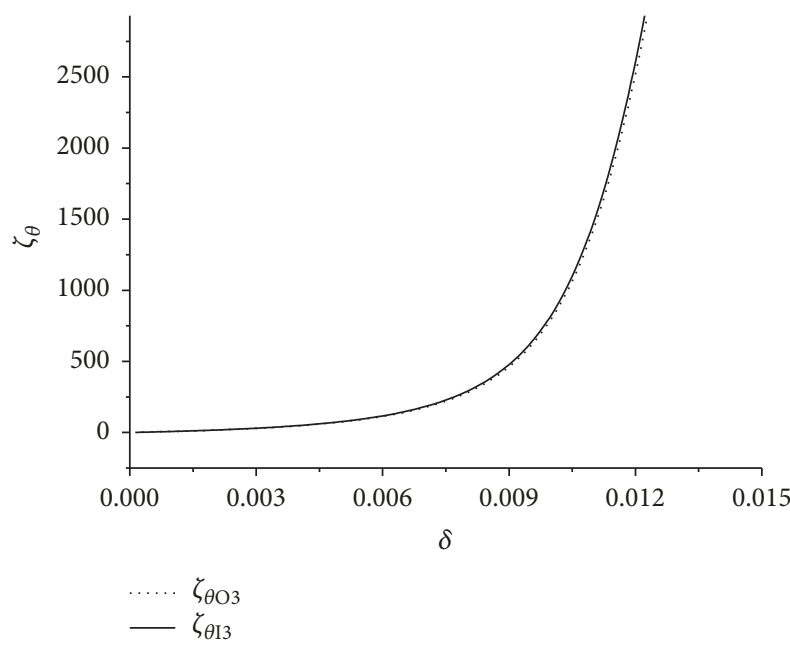

FIGURE 13: Effects of $\delta$ to $\zeta_{\theta \mathrm{II}}$ and $\zeta_{\theta \mathrm{O} 3}$ of adapter.

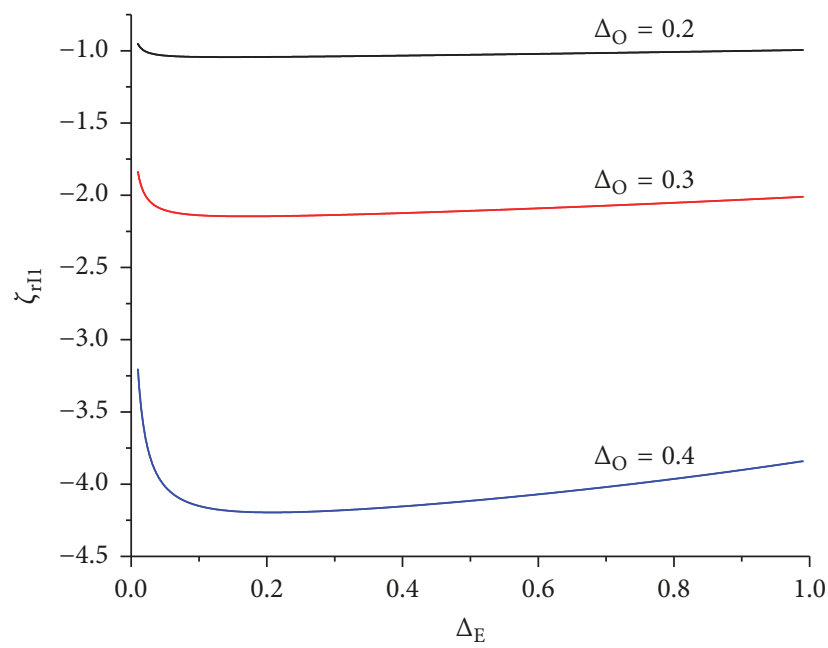

FIGURE 14: Effect curve of $\Delta_{\mathrm{E}}$ to $\zeta_{\text {rI1 }}$.

defined as $\Delta_{E}=T_{E}\left(T_{R}+T_{E}\right)$, where $T_{E}$ is radial thickness of sponge layer and $T_{R}$ is the radial thickness of the rubber. $\Delta_{O}=$ $T_{O} / T_{S}$ is defined as the ratio of precompression of adapter to sponge layer's thickness, $T_{O}$ is the precompression of adapter, and $T_{S}=T_{R}+T_{E}$. When $\Delta_{\mathrm{O}}=0.2,0.3$, and 0.4, Figures 14-25 are, respectively, the effect curves of thickness ratio $\Delta_{\mathrm{E}}$ to the radial stress of the inner sponge surface $\left(\zeta_{\mathrm{r} I 1}\right)$, the tangential stress of inner sponge $\left(\zeta_{\theta I 1}\right)$, the radial displacement of outer sponge surface $\left(\psi_{\mathrm{rM}}\right)$, the radial stress of outer sponge surface $\left(\zeta_{\mathrm{rM}}\right)$, the tangential stress of outer sponge surface $\left(\zeta_{\left.{ }_{\mathrm{MM}}\right)}\right)$, the tangential stress of inner rubber surface $\left(\zeta_{\theta \mathrm{M} 2}\right)$, the radial stress of outer rubber surface $\left(\zeta_{\mathrm{rO} 2}\right)$, the tangential stress of the outer rubber surface $\left(\zeta_{\theta \mathrm{O} 2}\right)$, the radial displacement of the inner launcher surface $\left(\psi_{\mathrm{r} 33}\right)$, the tangential stress of the inner launcher surface $\left(\zeta_{\theta \mathrm{II}}\right)$, the radial displacement of outer launcher surface $\left(\psi_{\mathrm{rO} 3}\right)$, and tangential stress of outer surface $\left(\zeta_{\theta \mathrm{O} 3}\right)$.

As shown in Figures 14, 15, 18, 20, and 20-25, the absolute values of stresses $\zeta_{\mathrm{rI} 1}, \zeta_{\theta \mathrm{I} 1}, \zeta_{\mathrm{rM}}, \zeta_{\mathrm{rO} 2}, \psi_{\mathrm{rI} 3}, \zeta_{\mathrm{\theta I} 3}, \psi_{\mathrm{rO} 3}$, and $\zeta_{\theta \mathrm{O} 3}$

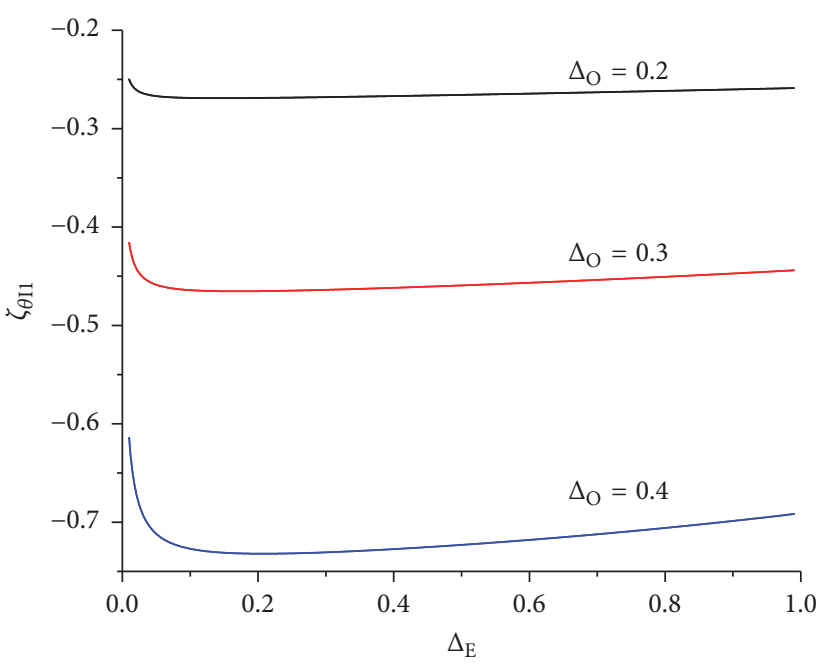

FIgURE 15: Effect curve of $\Delta_{\mathrm{E}}$ to $\zeta_{\theta I 1}$.

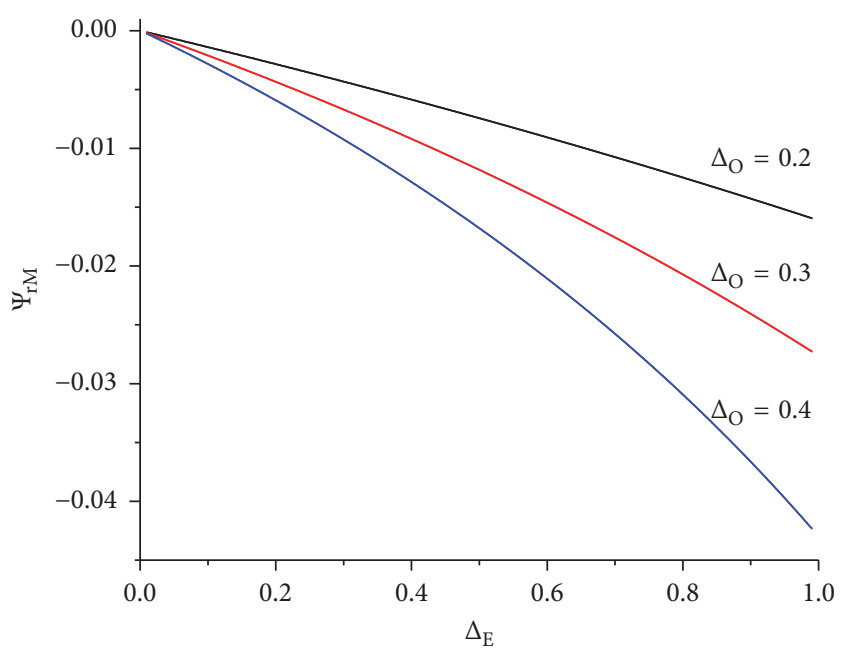

Figure 16: Effect curve of $\Delta_{\mathrm{E}}$ to $\psi_{\mathrm{rM}}$.

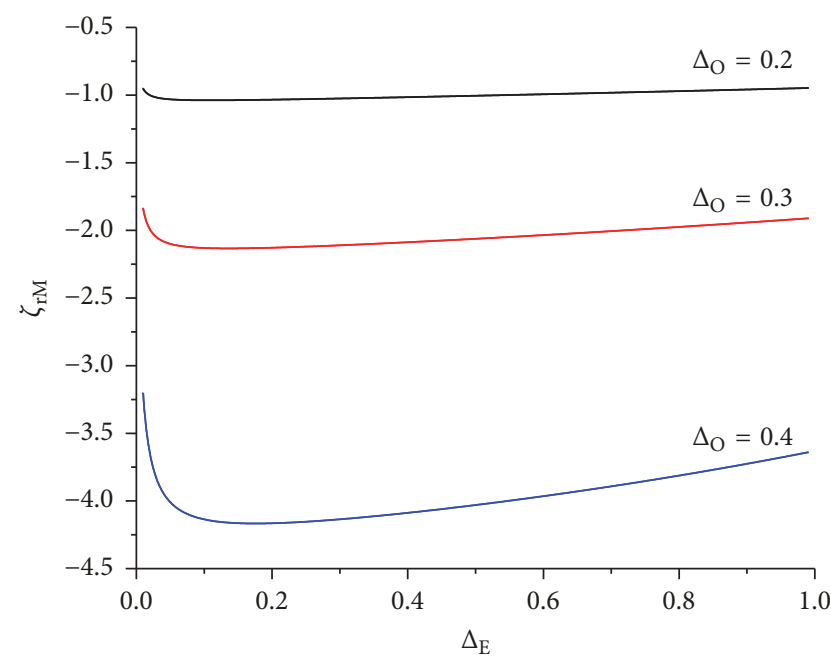

Figure 17: Effect curve of $\Delta_{\mathrm{E}}$ to $\zeta_{\mathrm{rM}}$. 


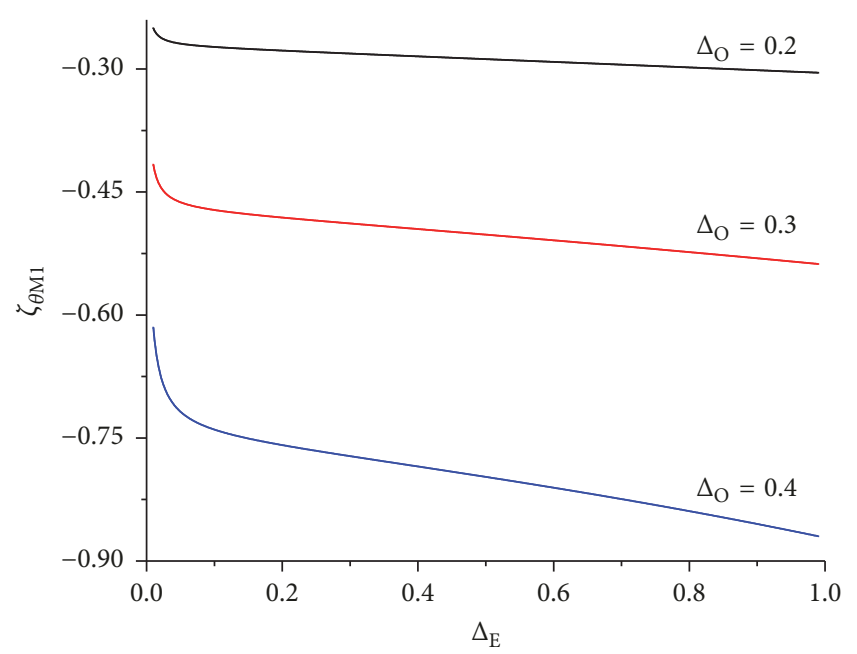

Figure 18: Effect curve of $\Delta_{\mathrm{E}}$ to $\zeta_{\theta \mathrm{M} 1}$.

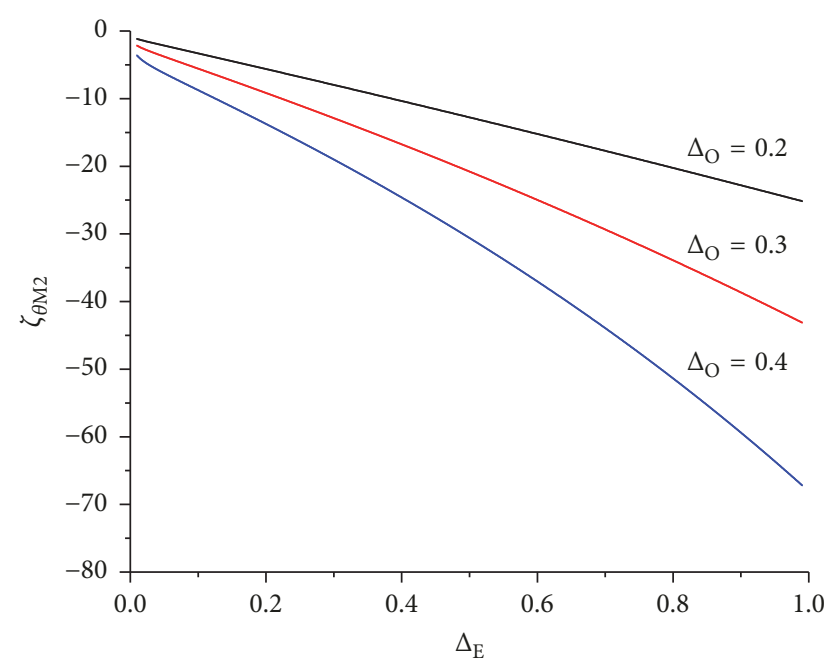

Figure 19: Effect curve of $\Delta_{\mathrm{E}}$ to $\zeta_{\theta \mathrm{M} 2}$.

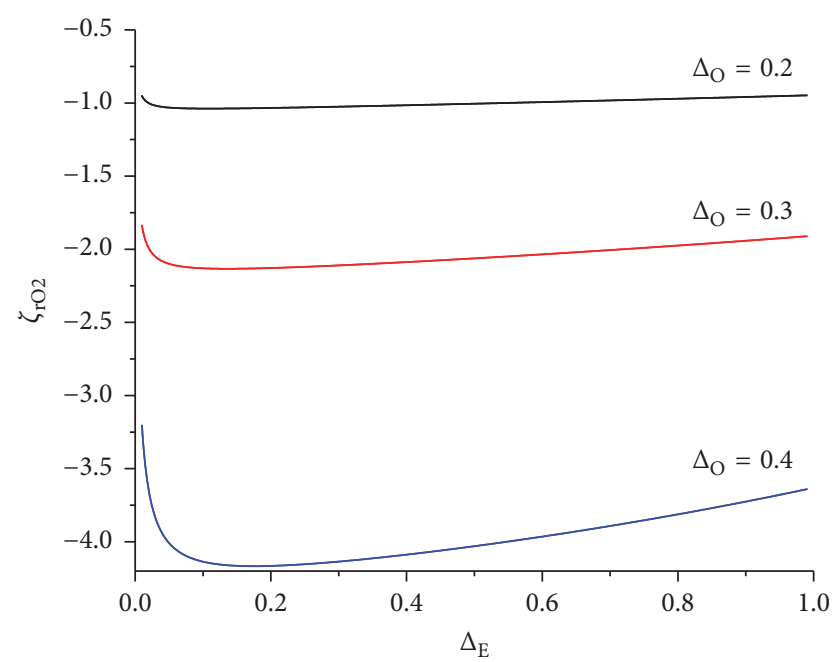

Figure 20: Effect curve of $\Delta_{\mathrm{E}}$ to $\zeta_{\mathrm{rO} 2}$.

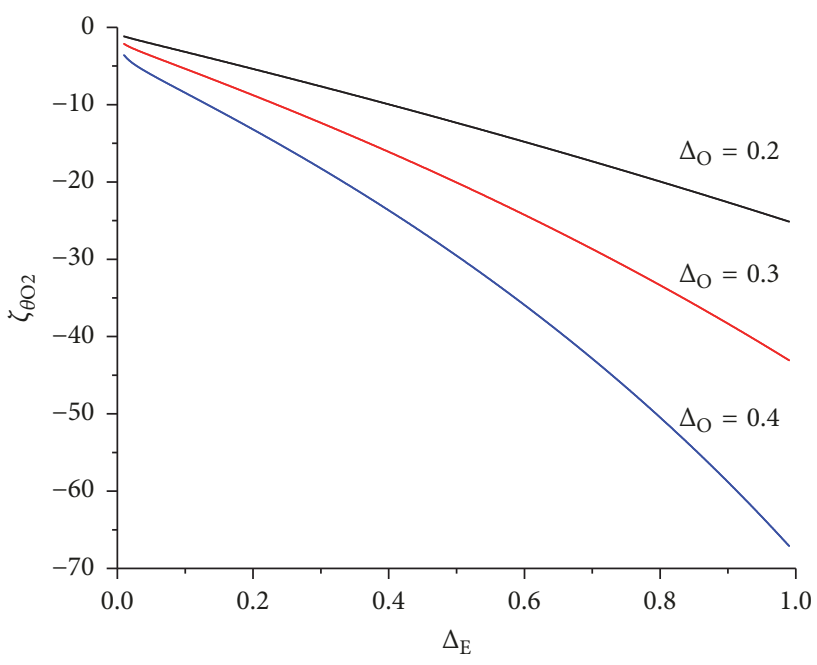

Figure 21: Effect curve of $\Delta_{\mathrm{E}}$ to $\zeta_{\mathrm{\theta O} 2}$.

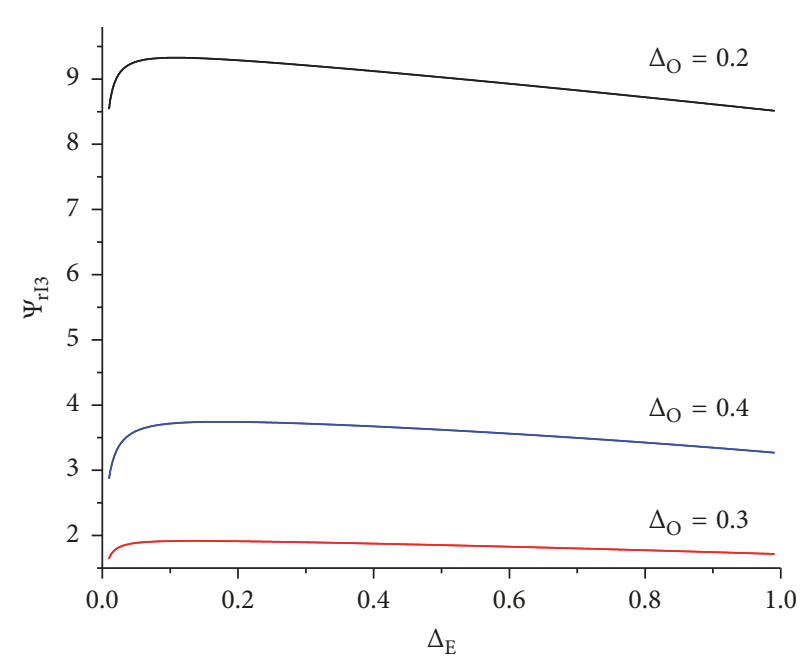

FIgURE 22: Effect curve of $\Delta_{\mathrm{E}}$ to $\psi_{\mathrm{rI} 3}$.

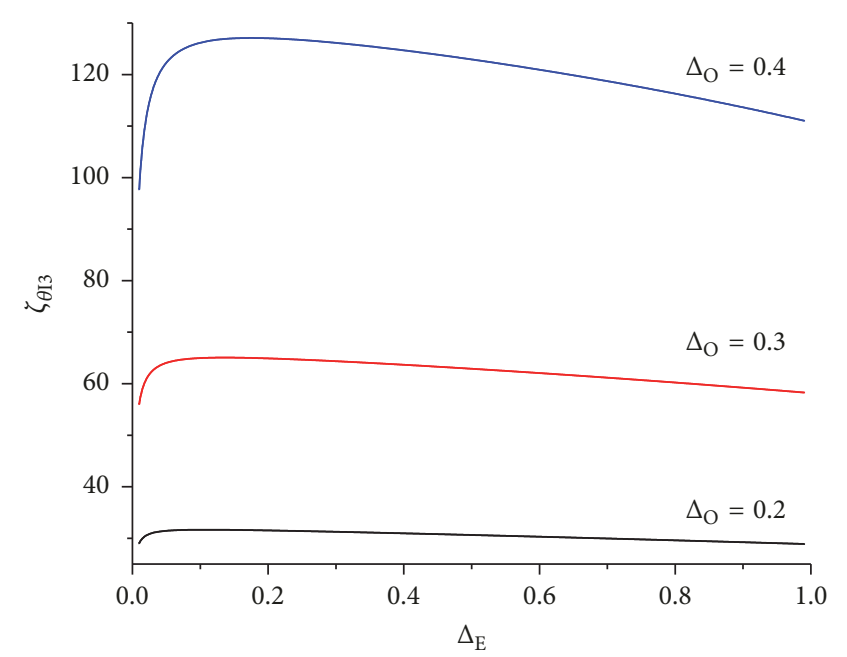

Figure 23: Effect curve of $\Delta_{\mathrm{E}}$ to $\zeta_{\theta \mathrm{I} 3}$. 


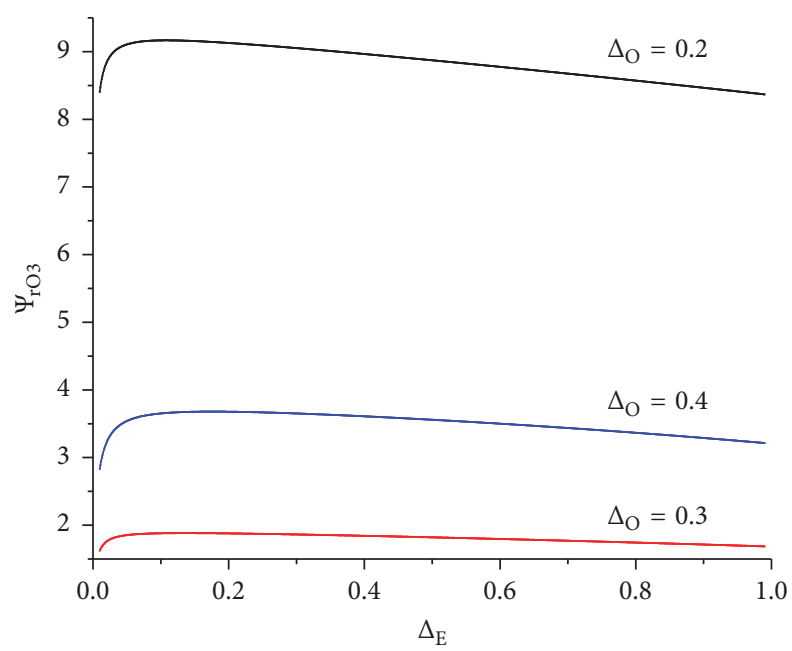

FIgURE 24: Effect curve of $\Delta_{\mathrm{E}}$ to $\psi_{\mathrm{rO} 3}$.

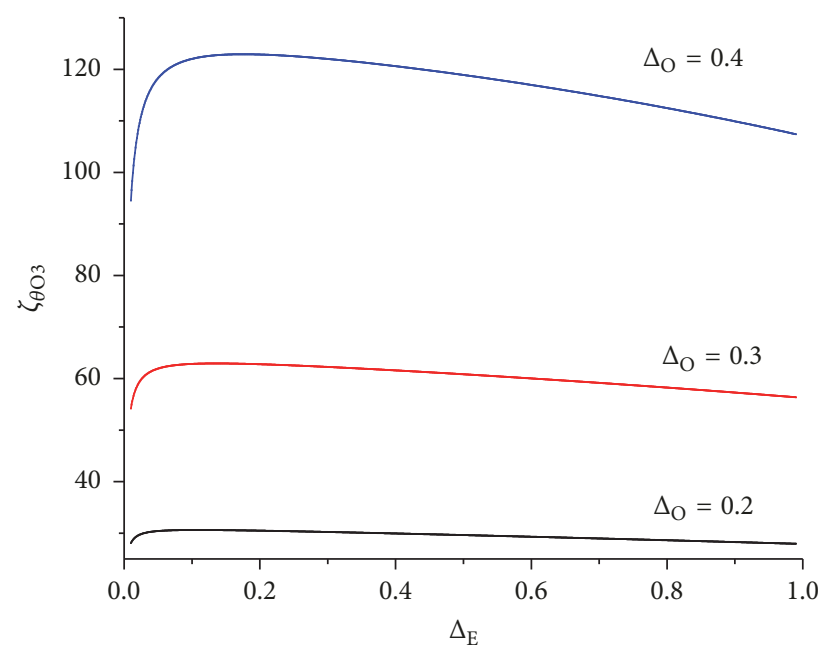

Figure 25: Effect curve of $\Delta_{\mathrm{E}}$ to $\zeta_{\theta \mathrm{O} 3}$.

change with $\Delta_{E}$, which first increase and then decrease with the maximum extremum point. When $\Delta_{\mathrm{O}}=0.2,0.3,0.4$, the horizontal ordinates of $\zeta_{\mathrm{rI} 1}$ and $\zeta_{\theta \mathrm{I} 1}$ with the maximum value are, respectively, 0.14499, 0.16984, and 0.20795. The horizontal ordinates of the corresponding maximum point of $\zeta_{\mathrm{rM}}, \zeta_{\mathrm{rO} 2}, \psi_{\mathrm{rI} 3}, \zeta_{\mathrm{\theta I} 3}, \psi_{\mathrm{rO} 3}$, and $\zeta_{\theta \mathrm{O} 3}$ are, respectively, 0.10936, 0.13422 , and 0.17482 . Furthermore, the horizontal ordinate of the maximum point is smaller than that of $\zeta_{\mathrm{rI} 1}$ and $\zeta_{\theta \mathrm{I} 1}$ and increases with the $\Delta_{\mathrm{O}}$. In view of Figures 16,19 , and $21, \psi_{\mathrm{rM}}$, $\zeta_{\theta \mathrm{M} 2}$, and $\zeta_{\theta \mathrm{O} 2}$ are approximately in proportion to $\Delta_{\mathrm{E}}$, when $\Delta_{E}>0.1, \zeta_{\theta \mathrm{M} 1}$ is approximately linear with $\Delta_{\mathrm{E}}$.

\section{Conclusions}

In this paper, the deformation and stress analytical formula of interference fit among the sponge cylinder, rubber cylinder, the adapter, and the launcher is deduced based on the assumption of axisymmetric plane. The deformation of adapter and the stress analytical solution are obtained and are verified by numerical simulation. The effects of precompression and the thickness ratio of sponge layer on the adapter's deformation and stress are studied. The following conclusions can be obtained.

(1) When the adapter carried out interference fit with launcher, the numerical simulation of radial displacement, radial stress, and tangential stress is consistent with that analytical solution, indicating that the derivation of the analytical formula for deformation and stress is correct.

(2) After the installation of adapter into the launcher, its outer surface moves inward and the displacement with the maximum absolute value appears on the adhesive surface. The rubber layer moves inward as a whole and each point in the launcher moves outward; the maximum displacement of the launcher is on the interference fit surface. For the sponge layer, the absolute value of the radial stress decreases from inside to outside, while for the rubber layer, the absolute value of the radial stress increases from inside to outside, and that of the launcher reduces from inside to outside until 0 . The three materials are all subjected to pressure. In terms of the tangential stress, that of the sponge layer increases along the radius while that of rubber layer decreases. The tangential stress of adapter is compressive stress while that of the launcher is tensile stress and decreases radially.

(3) When the precompression $\delta$ is smaller than 0.0075 or larger than $0.0105, \zeta_{\mathrm{rI} 1}, \zeta_{\mathrm{rM}}, \zeta_{\mathrm{\theta M} 2}, \zeta_{\theta \mathrm{O} 2}, \zeta_{\mathrm{rO} 2}, \psi_{\mathrm{rI} 3}, \psi_{\mathrm{rO} 3}, \zeta_{\theta \mathrm{I} 3}$, and $\zeta_{\mathrm{\theta O} 3}$ are approximately in proportion to precompression $\delta$. When $\delta$ is smaller than 0.0075 , the absolute value of corresponding stress and displacement increases slightly, while $\delta$ is larger than 0.0105 that increases sharply.

(4) The absolute values of stresses $\zeta_{\mathrm{rI} 1}, \zeta_{\theta \mathrm{I} 1}, \zeta_{\mathrm{rM}}, \zeta_{\mathrm{rO} 2}$, $\psi_{\mathrm{rI} 3}, \zeta_{\theta \mathrm{I} 3}, \psi_{\mathrm{rO} 3}$, and $\zeta_{\theta \mathrm{O} 3}$ vary with $\Delta_{\mathrm{E}}$, which increase firstly and then decrease with a extremum. The horizontal ordinate increases with $\Delta_{\mathrm{O}} \cdot \psi_{\mathrm{rM}}, \zeta_{\theta \mathrm{M} 2}$, and $\zeta_{\theta \mathrm{O} 2}$ are approximately in proportion with $\Delta_{\mathrm{E}}$, and when $\Delta_{E}>0.1, \zeta_{\theta \mathrm{M} 1}$ is approximately linear with $\Delta_{\mathrm{E}}$.

\section{Appendix}

The cylindrical coordinate system is adopted with the material coordinates of $(R, \Theta, Z)$ and the spatial coordinate of $(r, \theta, z)$. Because of the axisymmetric plane strain problem, the deformation mode can be set as

$$
\begin{aligned}
& r=f(R), \\
& \theta=\Theta, \\
& z=\lambda Z
\end{aligned}
$$

where $\lambda$ is the elongation of the cylinder in $z$ direction.

According to (A.1), the main elongation of the deformation gradient is

$$
\begin{aligned}
& \lambda_{r}=\frac{d r}{d R}, \\
& \lambda_{\theta}=\frac{r}{R}, \\
& \lambda_{z}=1
\end{aligned}
$$


Sponge is a hyperelastic compressible material whose strain energy function $W$ is a function of 3 main elongations; that is, $W=W\left(\lambda_{r}, \lambda_{\theta}, \lambda_{z}\right)$. The Cauchy stress in the 3 main elongation directions is

$$
\begin{aligned}
& \sigma_{r}=\frac{1}{\lambda_{\theta} \lambda_{z}} \frac{\partial W}{\partial \lambda_{r}}, \\
& \sigma_{\theta}=\frac{1}{\lambda_{r} \lambda_{z}} \frac{\partial W}{\partial \lambda_{\theta}}, \\
& \sigma_{z}=\frac{1}{\lambda_{r} \lambda_{\theta}} \frac{\partial W}{\partial \lambda_{z}}
\end{aligned}
$$

The axisymmetric plane strain problem should satisfy the static equilibrium equation with ignorance of gravity.

$$
\frac{d \sigma_{r}}{d r}+\frac{1}{r}\left(\sigma_{r}-\sigma_{\theta}\right)=0
$$

Equation (3) is substituted into (4):

$$
\begin{gathered}
-\frac{1}{\lambda_{\theta}^{2}} \frac{\partial \lambda_{\theta}}{\partial r} \frac{\partial W}{\partial \lambda_{r}}+\frac{1}{\lambda_{\theta}} \frac{\partial\left(\partial W / \partial \lambda_{r}\right)}{\partial r} \\
+\frac{1}{r}\left(\frac{1}{\lambda_{\theta}} \frac{\partial W}{\partial \lambda_{r}}-\frac{1}{\lambda_{r}} \frac{\partial W}{\partial \lambda_{\theta}}\right)=0
\end{gathered}
$$

From (A.2),

$$
\frac{\partial \lambda_{\theta}}{\partial r}=\frac{1}{R}-r \frac{1}{R^{2}} \frac{d R}{d r}
$$

Equation (A.6) is substituted into the equations (A.5):

$$
\begin{gathered}
-\frac{R^{2}}{r^{2}}\left(\frac{1}{R}-\frac{r}{R^{2}} \frac{\partial R}{\partial r}\right) \frac{\partial W}{\partial \lambda_{r}}+\frac{1}{\lambda_{\theta}} \frac{\partial\left(\partial W / \partial \lambda_{r}\right)}{\partial r} \\
+\frac{1}{r}\left(\frac{R}{r} \frac{\partial W}{\partial \lambda_{r}}-\frac{d R}{d r} \frac{\partial W}{\partial \lambda_{\theta}}\right)=0
\end{gathered}
$$

From (A.7),

$$
R \frac{\partial\left(\partial W / \partial \lambda_{r}\right)}{\partial r}+\frac{d R}{d r}\left(\frac{\partial W}{\partial \lambda_{r}}-\frac{\partial W}{\partial \lambda_{\theta}}\right)=0
$$

From (A.6),

$$
\begin{aligned}
R \frac{\partial\left(\partial W / \partial \lambda_{r}\right)}{\partial r} & =R\left(\frac{\partial^{2} W}{\partial \lambda_{r} \partial \lambda_{\theta}} \frac{\partial \lambda_{\theta}}{\partial r}+\frac{\partial^{2} W}{\partial \lambda_{r}^{2}} \frac{\partial \lambda_{r}}{\partial r}\right) \\
& =R \frac{\partial \lambda_{r}}{\partial r} \frac{\partial^{2} W}{\partial \lambda_{r}^{2}}+\frac{\partial^{2} W}{\partial \lambda_{r} \partial \lambda_{\theta}}\left(1-\frac{r}{R} \frac{d R}{d r}\right)
\end{aligned}
$$

Equation (A.9) is substituted into (A.8):

$$
\begin{aligned}
& R \frac{\partial \lambda_{r}}{\partial r} \lambda_{r} \frac{\partial^{2} W}{\partial \lambda_{r}^{2}}+\frac{\partial^{2} W}{\partial \lambda_{r} \partial \lambda_{\theta}}\left(\lambda_{r}-\lambda_{\theta}\right)+\frac{\partial W}{\partial \lambda_{r}}-\frac{\partial W}{\partial \lambda_{\theta}} \\
& \quad=0
\end{aligned}
$$

As $\partial \lambda_{r} / \partial r=\left(\partial \lambda_{r} / \partial R\right)(\partial R / \partial r)$, and $r=f(R)$ is the single valued function:

$$
\frac{\partial \lambda_{r}}{\partial r} \lambda_{r}=\frac{\partial \lambda_{r}}{\partial R} \frac{\partial R}{\partial r} \lambda_{r}=\frac{\partial \lambda_{r}}{\partial R} \frac{d R}{d r} \lambda_{r}=\frac{\partial \lambda_{r}}{\partial R}
$$

Equation (A.11) is substituted into (A.10); take $\lambda_{r}^{\prime}=$ $\partial \lambda_{r} / \partial R=d \lambda_{r} / d R$ into consideration:

$$
R \lambda_{r}^{\prime} \frac{\partial^{2} W}{\partial \lambda_{r}^{2}}+\frac{\partial^{2} W}{\partial \lambda_{r} \partial \lambda_{\theta}}\left(\lambda_{r}-\lambda_{\theta}\right)+\frac{\partial W}{\partial \lambda_{r}}-\frac{\partial W}{\partial \lambda_{\theta}}=0
$$

The Blatz-Ko strain energy function,

$$
\begin{aligned}
W & \left(\lambda_{r}, \lambda_{\theta}, \lambda_{z}\right) \\
& =\frac{\mu}{2}\left(\lambda_{r}^{-2}+\lambda_{\theta}^{-2}+\lambda_{z}^{-2}+2 \lambda_{r} \lambda_{\theta} \lambda_{z}-5\right)
\end{aligned}
$$

From (A.13),

$$
\begin{aligned}
\frac{\partial W}{\partial \lambda_{r}} & =\frac{\mu}{2}\left(-\frac{2}{\lambda_{r}^{3}}+2 \lambda_{\theta}\right) \\
\frac{\partial^{2} W}{\partial \lambda_{r}^{2}} & =\mu \frac{3}{\lambda_{r}^{4}} \\
\frac{\partial^{2} W}{\partial \lambda_{r} \partial \lambda_{\theta}} & =\mu \\
\frac{\partial W}{\partial \lambda_{\theta}} & =\frac{\mu}{2}\left(-\frac{2}{\lambda_{\theta}{ }^{3}}+2 \lambda_{r}\right)
\end{aligned}
$$

Equation (A.14) is substituted into (A.10):

$$
\begin{gathered}
R \lambda_{r}^{\prime} \frac{6}{\lambda_{r}^{4}}+2\left(\lambda_{r}-\lambda_{\theta}\right)+\left(-\frac{2}{\lambda_{r}^{3}}+2 \lambda_{\theta}\right) \\
-\left(-\frac{2}{\lambda_{r}^{3}}+2 \lambda_{r}\right)=0
\end{gathered}
$$

From (A.15),

$$
3 R \lambda_{r}^{\prime}+\left(\frac{\lambda_{r}^{4}}{\lambda_{\theta}^{3}}-\lambda_{r}\right)=0
$$

\section{Data Availability}

The data used to support the findings of this study are available from the corresponding author upon request.

\section{Conflicts of Interest}

The authors state that there are no conflicts of interest regarding the publication of this paper.

\section{Acknowledgments}

The authors gratefully acknowledge the financial supports from Innovation Partnership Fund for Universities of China Aerospace Science and Technology Corporation (CASC) and Natural Science Funds for Young Scholar of Jiangsu Province (no. BK 20170837). 


\section{References}

[1] K. Dominguez, "Methods of connecting testing equipment to a missile system," Tech. Rep. 9,739,568, U.S. Patent, 2017.

[2] A. B. Shelton, C. Martin, and W. A. Silva, "Computing aerodynamic damping of a generic missile with CFD," in Proceedings of the AIAA Aerospace Sciences Meeting, 2018.

[3] M.S. R. Chandra Murty, P. K. Sinha, and D. Chakraborty, "Effect of rocket exhaust of canisterized missile on adjoining launching system," Proceedings of the Institution of Mechanical Engineers, Part G: Journal of Aerospace Engineering, vol. 231, no. 11, pp. 2085-2097, 2017.

[4] S. Irish Angelin, S. Raja, N. Rajesh et al., "Ap-plicability of canister for barraging missiles," International Review of Aerospace Engineering, vol. 2, no. 6, pp. 86-90, 2015.

[5] B. Koohbor, A. Kidane, and W.-Y. Lu, "Characterizing the constitutive response and energy absorption of rigid polymeric foams subjected to intermediate-velocity impact," Polymer Testing, vol. 54, pp. 48-58, 2016.

[6] B. Su, Z. Zhou, G. Xiao, Z. Wang, X. Shu, and Z. Li, "A pressure-dependent phenomenological constitutive model for transversely isotropic foams," International Journal of Mechanical Sciences, vol. 120, pp. 237-248, 2017.

[7] C. Beckmann and J. Hohe, "A probabilistic constitutive model for closed-cell foams," Mechanics of Materials, vol. 96, pp. 96$105,2016$.

[8] G. Liang and K. Chandrashekhara, "Neural network based constitutive model for elastomeric foams," Engineering Structures, vol. 30, no. 7, pp. 2002-2011, 2008.

[9] D. Hao, D. Li, and Y. Liao, "A finite viscoelastic constitutive model for filled rubber-like materials," International Journal of Solids and Structures, vol. 64, pp. 232-245, 2015.

[10] J. Chen, M. Huang, and X. Wang, "Typical constitutive models of rubber materials and their ranges of application," International Materials Reviews, 2015.

[11] H. S. Lee, J. K. Shin, S. Msolli, and H. S. Kim, "Prediction of the dynamic equivalent stiffness for a rubber bushing using the finite element method and empirical modeling," International Journal of Mechanics and Materials in Design, pp. 1-15, 2017.

[12] N. Zhang, N. Hoang, and H. Du, "A novel dynamic absorber using enhanced magnetorheological elastomers for powertrain vibration control," Advanced Materials Research, vol. 47-50, pp. 117-120, 2008.

[13] J. M. Horton and G. E. Tupholme, "Approximate radial stiffness of rubber bush mountings," Materials and Corrosion, vol. 27, no. 3, pp. 226-229, 2006.

[14] Y. Zhou, Z. Zhuang, and H. Kezhi, "The solutions of axiymmetric plane stress for a hyperelastic material interference problem," Engineering Mechanics, vol. 21, no. 6, pp. 72-75, 2004.

[15] Y. Anani and G. H. Rahimi, "Field equations and general solution for axisymmetric thick shell composed of functionally graded incompressible hyperelastic materials," International Journal of Mechanical Sciences, 2017.

[16] M. L. Ju, H. Jmal, R. Dupuis, and E. Aubry, "Visco-hyperelastic constitutive model for modeling the quasi-static behavior of polyurethane foam in large deformation," Polymer Engineering \& Science, vol. 55, no. 8, pp. 1795-1804, 2015.

[17] M. El Hamdaoui, J. Merodio, and R. W. Ogden, "Loss of ellipticity in the combined helical, axial and radial elastic deformations of a fibre-reinforced circular cylindrical tube," International Journal of Solids and Structures, vol. 63, pp. 99108, 2015.
[18] E. Kirkinis, R. W. Ogden, and D. M. Haughton, "Some solutions for a compressible isotropic elastic material," Journal of Applied Mathematics and Physics, vol. 55, no. 1, pp. 136-158, 2004.

[19] Q. Zhang and J. A. Blume, "Surface wrinkling in generalized Blatz-Ko materials," Extreme Mechanics Letters, vol. 11, pp. 6876, 2017.

[20] H. Guo, W. Guo, and A. V. Amirkhizi, "Constitutive modeling of the tensile and compressive deformation behavior of polyurea over a wide range of strain rates," Construction and Building Materials, vol. 150, pp. 851-859, 2017.

[21] H. Shokrollahi, F. Fallah, and M. H. Kargarnovin, "An approach in deformation and stress analysis of elasto-plastic sandwich cylindrical shell panels based on harmonic differential quadrature method," Journal of Sandwich Structures and Materials, vol. 19, no. 2, pp. 167-191, 2017.

[22] Z. Yang, Y. Liu, B. Tian, and Y. Zhang, "Hot deformation and dynamic recrystallization of $\mathrm{TiC}(30 \mathrm{vol} \%) / \mathrm{Cu}-\mathrm{Al} 2 \mathrm{O} 3$ composites," Acta Materiae Compositae Sinica, vol. 32, no. 1, pp. 117-124, 2015.

[23] S. G. Cho, W. K. Park, and S. M. Yun, "Finite element analysis of lead rubber bearing by using strain energy function of hyperelastic material," Journal of The Korean Society of Civil Engineers, vol. 36, no. 3, pp. 361-374, 2016.

[24] M. Bahreman and H. Darijani, "New polynomial strain energy function; application to rubbery circular cylinders under finite extension and torsion," Journal of Applied Polymer Science, vol. 132, no. 13, 2015.

[25] N. Perez, "Theory of elasticity," in Fracture Mechanics, Springer International Publishing, 2017.

[26] K. Gou, M. Mallikarjuna, K. R. Rajagopal, and J. R. Walton, "Modeling fracture in the context of a strain-limiting theory of elasticity: a single plane-strain crack," International Journal of Engineering Science, vol. 88, pp. 73-82, 2015.

[27] R. Ansari, A. Shahabodini, and M. Faghih Shojaei, "Nonlocal three-dimensional theory of elasticity with application to free vibration of functionally graded nanoplates on elastic foundations," Physica E: Low-dimensional Systems and Nanostructures, vol. 76, pp. 70-81, 2016.

[28] A. Pourasghar and Z. Chen, "Thermoelastic response of CNT reinforced cylindrical panel resting on elastic foundation using theory of elasticity," Composites Part B: Engineering, vol. 99, pp. 436-444, 2016.

[29] R. McGill and P. Kenneth, "Solution of variational problems by means of a generalized newton-raphson operator," Aiaa Journal, vol. 2, no. 10, p. 28, 2015.

[30] W. Nazeer, A. Naseem, S. M. Kang, and Y. C. Kwund, "Generalized Newton Raphson's method free from second derivative," Journal of Nonlinear Science and Applications, vol. 9, no. 5, pp. 2823-2831, 2016. 


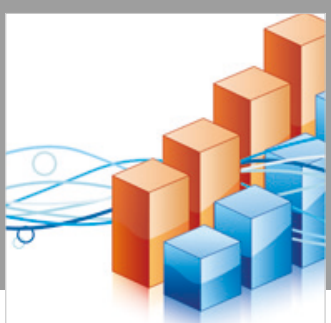

Advances in

Operations Research

\section{-n-m}
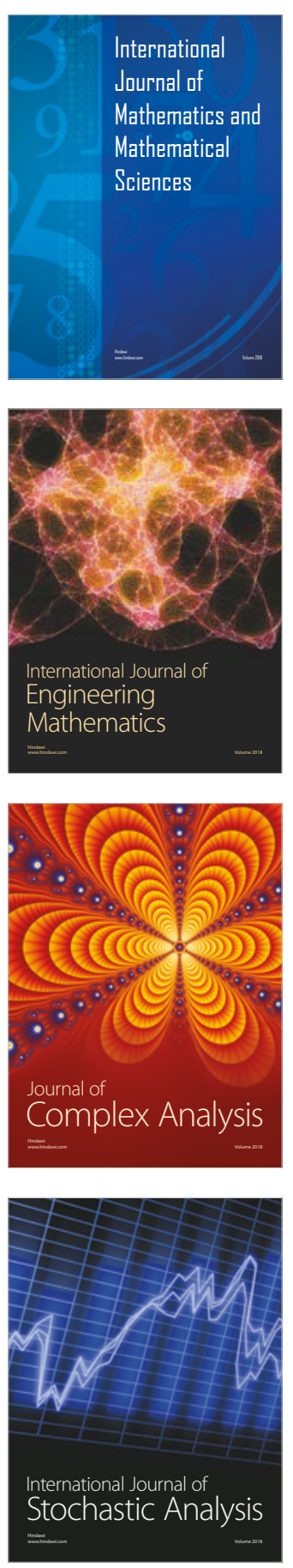
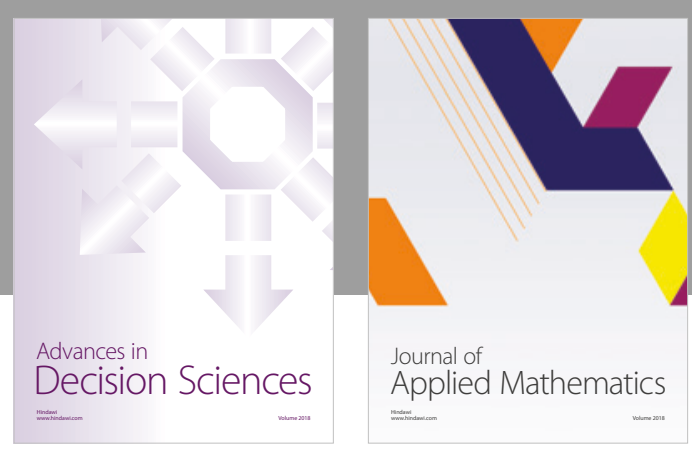

Journal of

Applied Mathematics
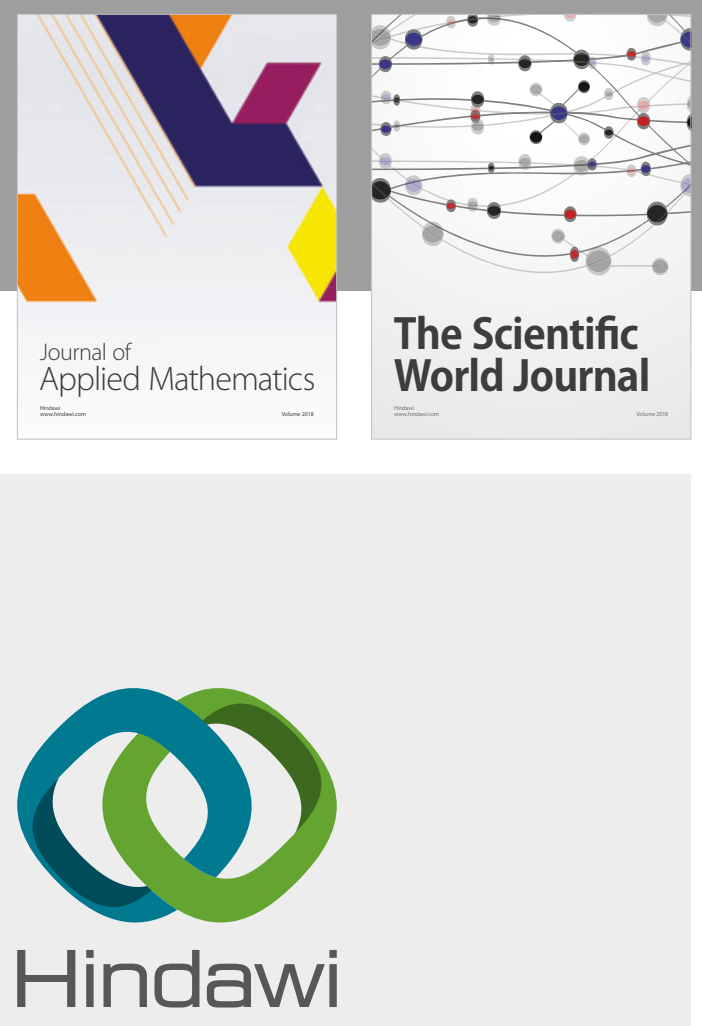

Submit your manuscripts at

www.hindawi.com

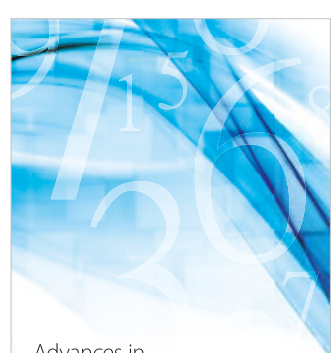

Advances in
Numerical Analysis
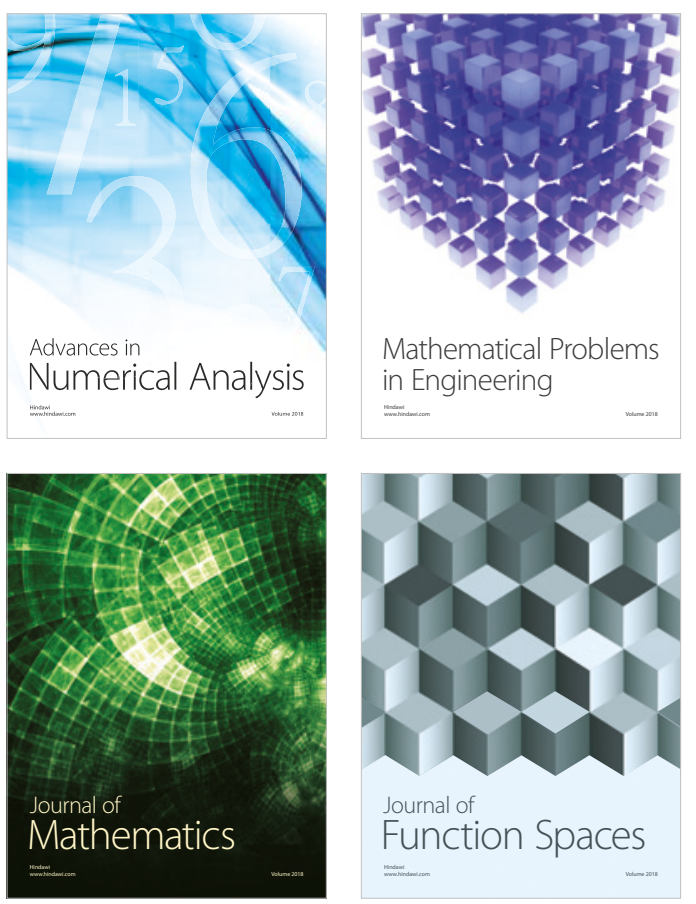

Mathematical Problems in Engineering

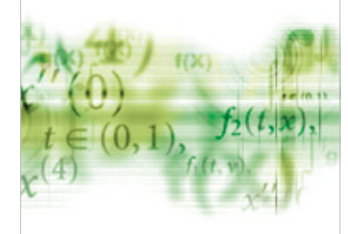

International Journal of

Differential Equations

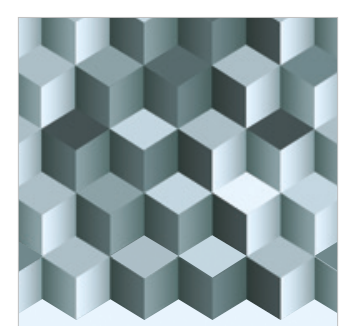

Journal of

Function Spaces

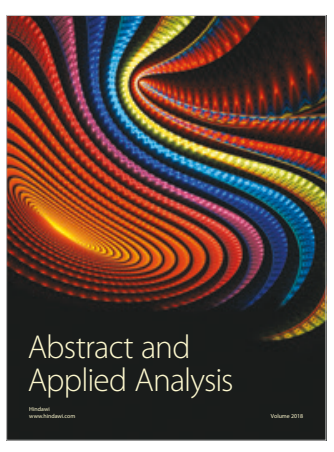

The Scientific

World Journal

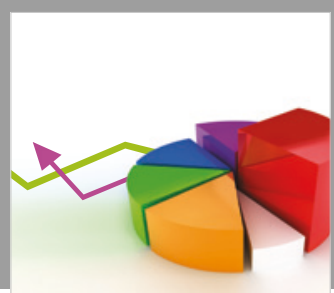

Journal of

Probability and Statistics
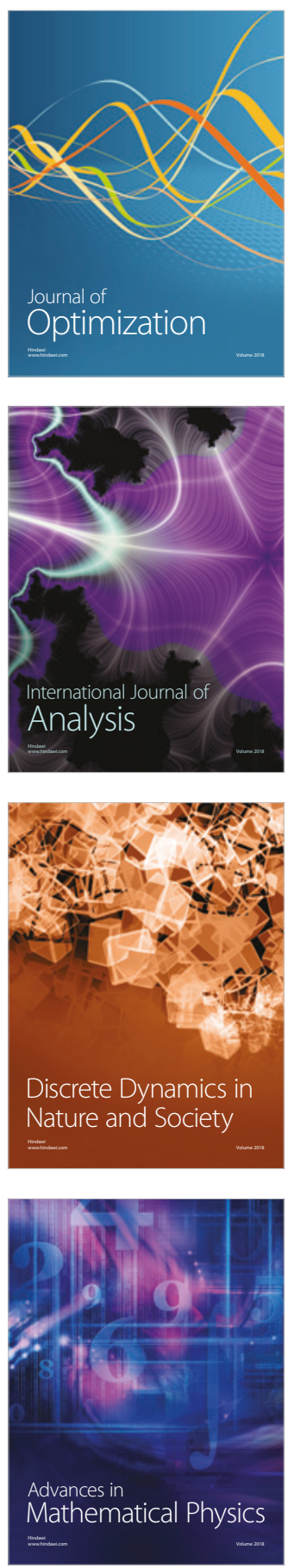